\title{
A Monte Carlo Based Analysis of Optimal Design Criteria
}

\author{
H. T. Banks, Kathleen J. Holm and Franz Kappel \\ Center for Quantitative Sciences in Biomedicine \\ Center for Research in Scientific Computation \\ North Carolina State University \\ Raleigh, NC 27695-8212 \\ and \\ Institute for Mathematics and Scientific Computation \\ University of Graz \\ Graz, Austria A8010
}

November 9, 2011

\begin{abstract}
Optimal design methods (designed to choose optimal sampling distributions through minimization of a specific cost function related to the resulting error in parameter estimates) for inverse or parameter estimation problems are considered. We compare a recent design criteria, $S E$-optimal design (standard error optimal design [8]) with the more traditional $D$-optimal and $E$-optimal designs. The optimal sampling distributions from each design are used to compute and compare standard errors; here the standard errors for parameters are computed using the optimal mesh along with Monte Carlo simulations as compared to asymptotic theory based standard errors. We illustrate ideas with two examples: the Verhulst-Pearl logistic population model [10] and the standard harmonic oscillator model [10].
\end{abstract}

AMS Subject Classification: 93B51, 62B10, 62B15, 62G08, 62H12, 90C31, 65C05.

Key Words: Optimal design methods, Least squares inverse problems, Fisher Information matrix, D-optimal, E-optimal, SE-optimal, Monte Carlo analysis. 


\section{Introduction}

In recent years modeling in the life sciences involves increasingly sophisticated models with a plethora of parameters to be estimated. This is due in part to increasingly stringent requirements concerning accuracy and the desire for adaptation of models to individual subjects. In contrast to this complex situation one usually is confronted with serious restrictions on data which often need to be obtained by noninvasive or modestly invasive procedures. The validation of such models is, as a result, a very challenging problem. It is therefore necessary to design the data collection procedures very carefully in order to optimize the information content carried by the data obtained. Methods of optimal experimental design are of central importance in this context and have justifiably received significant attention in the research literature (see for example [11, 14, 15, 22]). Since one has a number of different design criteria from which to choose, it is important to have procedures to compare typical results obtained by using different criteria. A natural criterion for comparison of different approaches are the standard errors of the parameters estimated on the basis of the data provided by the optimally designed experiments. One possibility which has been frequently employed is the asymptotic approximation of standard errors [23] which are based on only a single realization and the limiting approximations. However, such standard errors obtained in specific cases may not be sufficiently precise, because the underlying assumptions for these asymptotic approximations may not be satisfied. Therefore it is of interest to use a different approach which is not based on a single realization of the observation process, but uses a large number of realizations, with, of course, increased computational costs. To illustrate an alternative approach, we present in this paper a detailed Monte Carlo analysis of the performance of several optimal design methods for the logistic and harmonic oscillator examples which were previously [8] investigated using asymptotic standard errors as a measure of comparison.

\section{Optimal design of sampling times}

Given the duration or time interval $[0, T]$ for an experiment and the number $N$ of the sampling times allowed, the remaining fundamental question of optimal experimental design to be answered is the location of the sampling times in $[0, T]$. In [7] a general theoretical framework for optimal experimental design problems has been developed, which incorporates all design criteria based on the Fisher Information Matrix (FIM) (see (2.6) below for a definition of the FIM in case of discrete sampling times) for the given parameter estimation problem. This general theoretical framework is based on abstract sampling distributions which can be represented using probability measures on the Borel subsets of $[0, T]$, which typically cannot be realized by concrete measurement procedures. However, this general approach provides an abstract setting for existence and approximation results based on the fact that the space $\mathcal{P}(0, T)$ of probability measures on $[0, T]$ supplied with the Prohorov metric (see $[2,12,16,21]$ ) is a compact and separable metric space (see [7, Section 4]). In particular, this theory permits development of computational methods using discrete measures with a finite number of atoms, which are precisely those corresponding to sampling procedures which can be realized. In the following we present the results only for this case and refer for the general theory to [7].

Underlying our considerations is a mathematical output model

$$
\eta(t)=f(t, \theta), \quad t \in[0, T], \theta \in \mathcal{A},
$$

where $\eta(t) \in \mathbb{R}$ is the scalar measurable or observable output of the system, $f:[0, T] \times \mathcal{A} \rightarrow \mathbb{R}$ is a sufficiently smooth mapping, $\theta \in \mathbb{R}^{p}$ is the vector of system parameters, $\mathcal{A} \subset \mathbb{R}^{p}$ is the set of admissible parameter vectors and $T$ is the endpoint of the observation interval. 
To consider measures of uncertainty in estimated parameters, one also requires a statistical model [4]. Our statistical model is given by the stochastic observation process

$$
Y(t)=f\left(t, \theta_{0}\right)+\mathcal{E}(t), \quad t \in[0, T] .
$$

Here $\mathcal{E}$ is a stochastic process representing measurement errors and, as usual in statistical formulations $[4,7,13,23], \theta_{0}$ is the vector of the hypothesized "true" or nominal values of the unknown parameters. We make the following standard assumptions on the random variables $\mathcal{E}(t)$ :

$$
\begin{aligned}
\mathrm{E}(\mathcal{E}(t)) & =0, \quad t \in[0, T], \\
\operatorname{Var} \mathcal{E}(t) & =\sigma(t)^{2} I, \quad t \in[0, T], \\
\operatorname{Cov}(\mathcal{E}(t) \mathcal{E}(s)) & =\sigma(t)^{2} I \delta(t-s), \quad t, s \in[0, T],
\end{aligned}
$$

where $\delta(s)=1$ for $s=0$ and $\delta(s)=0$ for $s \neq 0$. A realization of the observation process is given by

$$
y(t)=f\left(t, \theta_{0}\right)+\epsilon(t), \quad t \in[0, T]
$$

where $y(t)$ is a concrete measurement for $\eta(t)$ at time $t$ and the measurement error $\epsilon(t)$ is a realization of $\mathcal{E}(t)$.

The statistical model we assume is a classical first model widely found in regression analysis [13, 23] and is often employed in development of ideas (asymptotic distribution theory, statistical comparison tests, etc.) as well as in certain applications. Two (the second and third) of the assumptions (2.3) are sometimes violated in repeated measurement data problems, especially in economic data and in biological data with high frequency sampling using model response dependent assays. The assumptions of independence and zero correlation for the statistical model can often be checked/supported via residual plots $[4,10,13,23]$ (after carrying out the inverse or regression problem fits with ordinary least squares). More sophisticated error models (e.g., nonconstant variance, nontrivial correlation) might also require different inverse problem formulations [4] (e.g., generalized least squares or maximum likelihood if sufficient information is known about the distributions of errors). Our choice of this simple statistical model was motivated primarily for two reasons: (i) it is simple, widely known/used and easy to use in comparison of designs, and (ii) our own efforts (e.g., see [3, 5, 6, 9, 10] and the references therein) in numerous inverse problems with longitudinal data for HIV patients, data for insect and marine populations, and vibrational engineering data often have dealt with data in which independence of sampling appeared to be reasonable. Even when correlation might be present, it is often assumed fast decaying with respect to distance/time between observations (this is a standard correlation model-see $[13,23]$ ) and independence is a reasonable assumption for low frequency sampling times. However, there are numerous situations in practice where independence is readily violated (e.g., economics and biology) and techniques in the presence of nontrivial data correlations are an active area of optimal design research (see $[19,20,24]$ and the references therein).

For sampling points $\tau=\left\{t_{i}\right\}_{i=1, \ldots, N}, t_{1} \leq \cdots \leq t_{N}$ in $[0, T]$, we introduce the weighted least squares criterion

$$
J(y, \theta)=\sum_{i=1}^{N} \frac{1}{\sigma\left(t_{i}\right)^{2}}\left(y\left(t_{i}\right)-f\left(t_{i}, \theta\right)\right)^{2}, \quad \theta \in \mathcal{A} .
$$

We seek the parameter estimate $\hat{\theta}$ by minimizing $J(y, \theta)$ for $\theta \in \mathcal{A}$,

$$
\hat{\theta}=\underset{\theta \in \mathcal{A}}{\operatorname{argmin}} J(y, \theta) \text {. }
$$

Of central importance for our discussions is the Fisher Information Matrix (FIM),

$$
F\left(\tau, \theta_{0}\right)=\sum_{j=1}^{N} \frac{1}{\sigma^{2}\left(t_{j}\right)} \nabla_{\theta} f\left(t_{j}, \theta_{0}\right)^{\top} \nabla_{\theta} f\left(t_{j}, \theta_{0}\right),
$$


where $\nabla_{\theta}$ is a row vector given by $\left(\partial_{\theta_{1}}, \ldots, \partial_{\theta_{p}}\right)$ and hence $\left.\nabla_{\theta} f\left(t_{j}, \theta\right)\right|_{\theta=\theta_{0}}$, the gradient of $f$ with respect to $\theta$, is a row vector of length $p$.

The estimate $\hat{\theta}$ obtained in (2.5) for a specific set of measurements is a representation of a random variable $\hat{\Theta}=\hat{\Theta}\left(\tau, \theta_{0}\right)$, the least squares estimator for our parameter estimation problem. We are interested in the statistical properties of $\hat{\Theta}$. Using linearization of $f(t, \theta)$ around $\theta_{0}$ and asymptotic results of statistical analysis for least squares estimation we obtain that (see [7, Appendix])

$$
\begin{aligned}
\mathrm{E}(\hat{\Theta}) & \approx \theta_{0}, \\
\operatorname{Cov}(\hat{\Theta}) & \approx F\left(\tau, \theta_{0}\right)^{-1} .
\end{aligned}
$$

As a consequence we have the following asymptotic standard errors for the components of the estimate $\hat{\theta}$ obtained in (2.5):

$$
S E\left(\hat{\theta}_{k}\right)=\sqrt{\left(F\left(\tau, \theta_{0}\right)^{-1}\right)_{k k}}, \quad k=1, \ldots, p .
$$

The major question in optimal design of experiments which we consider in this paper is how to best choose $\tau=\left\{t_{i}\right\}_{i=1, \ldots, N} \in \mathcal{T}$, where $\mathcal{T}$ is the set of all time meshes such that $0 \leq t_{1} \leq \cdots \leq t_{i} \leq$ $t_{i+1} \leq \cdots \leq t_{N} \leq T$. In order to characterize "best" we have to use a design criterion. In order to define such design criteria we observe that the FIM $F\left(\tau, \theta_{0}\right)$ depends critically on the sampling mesh $\tau$. Let a continuous functional $\mathcal{J}: \mathbb{R}^{p \times p} \rightarrow \mathbb{R}^{+}$be given. The optimal design problem associated with $\mathcal{J}$ is the one of finding a sampling mesh $\tau^{*} \in \mathcal{T}$ such that

$$
\mathcal{J}\left(F\left(\tau^{*}, \theta_{0}\right)\right)=\min _{\tau \in \mathcal{T}} \mathcal{J}\left(F\left(\tau, \theta_{0}\right)\right) .
$$

The mapping $\tau \rightarrow \mathcal{J}\left(F\left(\tau, \theta_{0}\right)\right)$ is the design criterion used for the design problem.

The formulation (2.8) incorporates all optimal design methods which are based on minimizing or maximizing a functional depending continuously on the Fisher Information Matrix. In this paper we compare the results obtained with the following optimal design methods: $S E$-optimal design as introduced in [7], $D$-optimal design and $E$-optimal design. The design cost functional $\mathcal{J}$ for the $S E$-optimal design method (see [7]) is given by

$$
\mathcal{J}_{S E}(F)=\sum_{i=1}^{p} \frac{1}{\theta_{0, i}^{2}}\left(F^{-1}\right)_{i i},
$$

where $F=F\left(\tau, \theta_{0}\right)$ is the FIM defined above in (2.6), $\theta_{0}$ is the true parameter vector, and $p$ is the number of parameters to be estimated. Note that both inversion and taking the trace of a matrix are continuous operations. We observe that $F\left(\tau, \theta_{0}\right)_{i i}^{-1}=S E\left(\hat{\theta}_{i}\right)^{2}$. Therefore, $S E$-optimal design minimizes the sum of squared normalized asymptotic standard errors.

$D$-optimal design minimizes the volume of the confidence ellipsoid for the asymptotic covariance matrix $F\left(\tau, \theta_{0}\right)^{-1}$. The design cost functional for $D$-optimal design (see $[11,15]$ ) is given by

$$
\mathcal{J}_{D}(F)=\operatorname{det} F^{-1} \text {. }
$$

Again we note that taking the determinant is a continuous operation on matrices so that $\mathcal{J}_{D}$ is continuous in $F$ as required by the theory.

$E$-optimal design minimizes the principle axis of the confidence ellipsoid of the covariance matrix (defined in the asymptotic theory summarized in the next section). The design cost functional for $E$-optimal design (see $[1,11]$ ) is given by

$$
\mathcal{J}_{E}(F)=\max _{i} \frac{1}{\lambda_{i}}
$$

where $\lambda_{i}, i=1 \ldots p$, are the eigenvalues of $F$ (which are continuous functions of $F$ ). Consequently $\frac{1}{\lambda_{i}}, i=1, \ldots, p$, are the eigenvalues of the asymptotic covariance matrix $F\left(\tau, \theta_{0}\right)^{-1}$. 


\subsection{Constrained optimization and implementations}

Each optimal design computational method we employ is based on constrained optimization to find the mesh of time points $\tau^{*}=\left\{t_{i}^{*}\right\}, i=1, \ldots, N$, that satisfy

$$
\mathcal{J}\left(F\left(\tau^{*}, \theta_{0}\right)\right)=\min _{\tau \in \mathcal{T}} \mathcal{J}\left(F\left(\tau, \theta_{0}\right)\right),
$$

where $\mathcal{T}$ is the set of all time meshes such that $0 \leq t_{1} \leq \cdots \leq t_{i} \leq t_{i+1} \leq \cdots \leq t_{N} \leq T$.

These optimal design methods were implemented using constrained optimization algorithms, either MATLAB's fmincon or SolvOpt, developed by A. Kuntsevich and F. Kappel [18, 17], with four variations of the constraint implementation. We denote by $(\mathrm{C} 1)-(\mathrm{C} 4)$ these different constraint implementations (which most often do result in different parameter and standard error outcomes even in cases where the $\left\{t_{i}\right\}_{i=1, \ldots, N}$ are initially required to satisfy similar constraints). Complete details of the precise implementation differences in the algorithms are given in the appendix of [8]. We briefly summarize these here.

(C1) In this case we impose the inequality constraints

$$
0 \leq t_{1} \leq \cdots \leq t_{i} \leq t_{i+1} \leq \cdots \leq t_{N} \leq T
$$

i.e., the optimal mesh may or may not contain 0 and $T$. In this case we optimize over $N$ variables.

(C2) In this case we fix $t_{1}=0, t_{N}=T$ and impose the inequality constraints

$$
0 \leq t_{2} \leq \cdots \leq t_{N-1} \leq T
$$

i.e., the optimal mesh contains 0 and $T$. Hence we optimize over $N-2$ variables.

(C3) We also fix $t_{1}=0, t_{N}=T$ and represent the other mesh points by $t_{i}=t_{i-1}+\nu_{i}, i=2, \ldots, N-1$. For $\nu_{i}, i=2, \ldots, N-1$ we impose the inequality constraints

$$
\nu_{i} \geq 0, i=2, \ldots, N-1 \text {, and } \nu_{2}+\cdots+\nu_{N-1} \leq T .
$$

Note that the optimal mesh always contains 0 and $T$ as we optimize over $N-2$ variables.

(C4) We fix $t_{1}=0$ and, as in case (C3), represent the other sampling times by $t_{i}=t_{i-1}+\nu_{i}$, $i=2, \ldots, N$. For $\nu_{i}, i=2, \ldots, N$, we impose the constraints

$$
\nu_{i} \geq 0, i=2, \ldots, N \text {, and } T=\nu_{2}+\cdots+\nu_{N} .
$$

The equality constraint can be also written as $\nu_{N}=T-\sum_{i=2}^{N-1} \nu_{i}$. The optimal mesh again contains 0 and $T$, and we optimize over $N-1$ variables. Note that in view of the equality constraint and the definition of the $\nu_{i}$ we have $t_{N}=\nu_{2}+\cdots+\nu_{N}=T$.

\section{Monte Carlo methodology}

For a single Monte Carlo trial, we generate simulated data on the optimal mesh $\tau^{*}=\left\{t_{j}\right\}_{j=1, \ldots, N}$ based on the true parameter vector $\theta_{0}$ corresponding to our statistical model

$$
y_{j}=f\left(t_{j}, \theta_{0}\right)+\epsilon_{j}, \quad j=1, \ldots, N,
$$


where the $\epsilon_{j}$ are realizations of $\mathcal{E}_{j} \sim \mathcal{N}\left(0, \sigma_{0}^{2}\right)$ for $j=1, \ldots, N$. Parameters are estimated using OLS taking the initial parameter guess to be $\theta^{0}=1.4 \theta_{0}$. The parameter estimates $\hat{\theta}$ are stored, and the process is repeated with new simulated data corresponding to the optimal mesh for $M$ Monte Carlo trials. For the concrete examples considered in Sections 4 and 5 we always take

$$
M=1000 .
$$

More trials increase the resolution though the results remain unchanged.

In addition, for each simulated data set we computed the estimated Fisher Information Matrix (FIM) $\hat{F}=F\left(\tau^{*}, \hat{\theta}\right)$, to determine if the $95 \%$ confidence ellipsoid contains the true parameter values $\theta_{0}$. The $95 \%$ confidence ellipsoid [23] corresponding to normally distributed residuals is defined by

$$
(\theta-\hat{\theta})^{\top} \hat{F}(\theta-\hat{\theta})=p \mathrm{~F}_{p, n-p}^{\alpha},
$$

where $\mathrm{F}_{p, n-p}^{\alpha}$ is the F-distribution with $p$ numerator degrees of freedom, $n-p$ denominator degrees of freedom, and critical level $\alpha=0.05$. The confidence ellipsoid will contain the true parameter vector $\theta_{0}$ if the following inequality holds:

$$
\left(\theta_{0}-\hat{\theta}\right)^{\top} \hat{F}\left(\theta_{0}-\hat{\theta}\right) \leq p \mathrm{~F}_{p, n-p}^{\alpha}
$$

The Monte Carlo analysis was carried out for the three different optimal design methods ( $S E$-optimal, $D$-Optimal and $E$-optimal) as well as for the uniform mesh, each time with $M$ new sets of simulated data. For each Monte Carlo analysis, the average parameter estimate $\hat{\theta}_{\text {avg }}$ is reported along with its standard deviation $\hat{\theta}_{\mathrm{SD}}$. We also will compare the standard deviations $\hat{\theta}_{\mathrm{SD}}$ of these Monte Carlo trials to the asymptotic standard errors $S E(\hat{\theta})$ as computed using asymptotic theory in (2.7).

In addition, we report the proportion $\hat{P}$ of confidence ellipsoids containing the true vector $\theta_{0}$ and the corresponding standard error $S E(\hat{P})$,

$$
\hat{P}=\frac{\# \text { of } 95 \% \text { confidence ellipsoids containing } \theta_{0}}{M}, \quad S E(\hat{P})=\sqrt{\frac{\hat{P}(1-\hat{P})}{M}} .
$$

Note that $\hat{P}$ is the estimate of $P_{0}$ the true proportion. Figure 1 gives an example of $95 \%$ confidence ellipsoids corresponding to parameter estimates for given simulated data. The true parameter vector is plotted as a point in $\mathbb{R}^{p}=\mathbb{R}^{2}$. Ellipsoids that contain the true parameter vector are gray and those that do not are black, as determined by the inequality given in (3.1). We are interested to see if $\hat{P}$ is close to 0.95 (95\% confidence ellipsoid) for the different optimal design methods and the uniform mesh. 


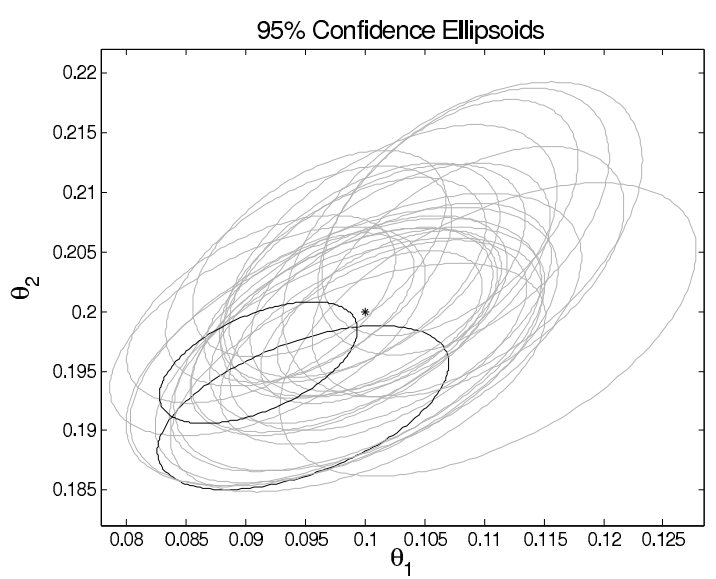

Figure 1: A generic example of $95 \%$ confidence ellipsoids (from $M=30$ simulated data sets, estimating $p=2$ parameters), where the true value is given by the star. Confidence ellipsoids containing the true value are gray, and confidence ellipsoids not containing the true value are black.

\section{Results for the logistic equation}

\subsection{The Verhulst-Pearl logistic population model}

The widely used logistic population model describes the growth of a population in an environment with a finite carrying capacity for the population. It is given by the differential equation:

$$
\dot{x}(t)=r x(t)(1-x(t) / K), \quad x(0)=x_{0},
$$

where $K$ is the carrying capacity of the environment for the population, $r$ is the intrinsic growth rate, and $x_{0}$ is the initial population size. The analytical solution to this initial value problem is given by

$$
x(t)=x(t, \theta)=\frac{K}{1+\left(K / x_{0}-1\right) e^{-r t}}, \quad t \geq 0,
$$

where $\theta=\left(K, r, x_{0}\right)^{\top}$ is the vector of system parameters.

For the statistical model in this case we choose

$$
f(t, \theta)=x(t, \theta), \quad t \geq 0 .
$$

In all our simulations we took the nominal parameter vector $\theta_{0}$ and the length $T$ of the sampling interval to be

$$
\theta_{0}=\left(K_{\text {nom }}, r_{\text {nom }}, x_{0, \text { nom }}\right)^{\top}=(17.5,0.7,0.1)^{\top}, \quad T=25 .
$$

\subsection{Monte Carlo analysis for the logistic equation}

Monte Carlo analysis was performed using the optimal design points found using constraint implementation (C2) and SolvOpt with $N=10$ or $N=15$ (see Figure 2). The nominal parameter vector $\theta_{0}$ and $T$ are always given by (4.2). The Monte Carlo analysis was conducted three times for each optimal design mesh taking different levels of constant variance $\left(\sigma^{2}(t)=\sigma_{0}^{2}\right.$ for all $\left.t\right)$ noise in the simulated data,

$$
\sigma_{0}^{2}=0.01,0.05 \text { or } 0.1
$$


Tables $1-3$ and $5-7$ contain the average estimates and standard deviations obtained from the Monte Carlo trials for $N=10$ and $N=15$ respectively and for the three different noise levels. In addition, the tables show the asymptotic standard errors, obtained from (2.7) using the true parameter values, for comparison with the standard deviations of the Monte Carlo parameter estimates. The percentage of $95 \%$ confidence ellipsoids which contain the true parameter vector and the corresponding standard errors as given by (3.2) are shown in Tables 4 and 8 . Histograms of the parameter estimates are plotted in Figures $3-5$ for $N=10$ and Figures $6-8$ for $N=15$. Each figure corresponds to a specific value of $\sigma_{0}^{2}$. Subfigures represent histograms for different parameter estimates, $\hat{\theta}=\left(\hat{K}, \hat{r}, \hat{x}_{0}\right)^{\top}$. Within each subfigure there are histograms for a specific parameter originating from an optimal design mesh or the uniform mesh.
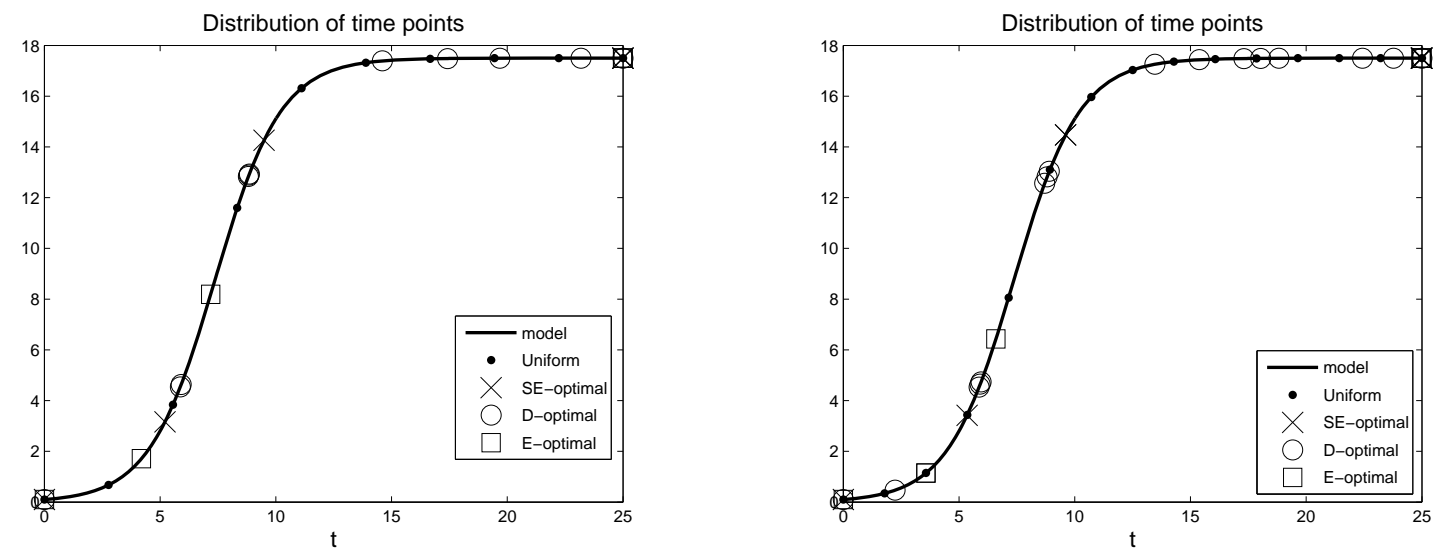

Figure 2: Optimal sampling times according to the optimal design criteria and uniform sampling times plotted on the logistic curve. Optimal sampling times were obtained using SolvOpt with $N=10$ (left panel), $N=15$ (right panel), and the constraint implementation (C2).

Table 1: Average estimates $\hat{\theta}_{\text {avg }}$ and corresponding standard deviations $\hat{\theta}_{\mathrm{SD}}$ from $M=1000$ Monte Carlo trials as well as asymptotic standard errors $S E(\hat{\theta})$.

\begin{tabular}{|c||c|c|c|c|}
\hline \multicolumn{5}{|c|}{$\boldsymbol{N}=\mathbf{1 0}, \boldsymbol{\sigma}_{\mathbf{0}}^{\mathbf{2}}=\mathbf{0 . 0 1}$} \\
\hline & $S E$-opt & $D$-opt & $E$-opt & Uniform \\
\hline \hline$\hat{K}_{\text {avg }}$ & 17.5019 & 17.5015 & 17.5013 & 17.5003 \\
\hline$\hat{K}_{\mathrm{SD}}$ & $4.527 \times 10^{-2}$ & $4.378 \times 10^{-2}$ & $3.495 \times 10^{-2}$ & $4.354 \times 10^{-2}$ \\
\hline$S E(\hat{K})$ & $4.466 \times 10^{-2}$ & $4.534 \times 10^{-2}$ & $3.535 \times 10^{-2}$ & $4.266 \times 10^{-2}$ \\
\hline$\hat{r}_{\text {avg }}$ & 0.7003 & 0.7004 & 0.7004 & 0.6995 \\
\hline$\hat{r}_{\mathrm{SD}}$ & $8.560 \times 10^{-3}$ & $9.341 \times 10^{-3}$ & $2.247 \times 10^{-2}$ & $1.198 \times 10^{-2}$ \\
\hline$S E(\hat{r})$ & $7.167 \times 10^{-3}$ & $7.673 \times 10^{-3}$ & $1.928 \times 10^{-2}$ & $1.005 \times 10^{-2}$ \\
\hline$\hat{x}_{0, \text { avg }}$ & 0.1000 & 0.1000 & 0.1009 & 0.1007 \\
\hline$\hat{x}_{0, \mathrm{SD}}$ & $6.087 \times 10^{-3}$ & $7.058 \times 10^{-3}$ & $1.577 \times 10^{-2}$ & $8.944 \times 10^{-3}$ \\
\hline$S E\left(\hat{x}_{0}\right)$ & $4.297 \times 10^{-3}$ & $4.965 \times 10^{-3}$ & $9.996 \times 10^{-3}$ & $6.194 \times 10^{-3}$ \\
\hline
\end{tabular}


Table 2: Average estimates $\hat{\theta}_{\mathrm{avg}}$ and corresponding standard deviations $\hat{\theta}_{\mathrm{SD}}$ from $M=1000$ Monte Carlo trials as well as asymptotic standard errors $S E(\hat{\theta})$.

\begin{tabular}{|c||c|c|c|c|}
\hline \multicolumn{5}{|c|}{$\boldsymbol{N}=\mathbf{1 0}, \boldsymbol{\sigma}_{\mathbf{0}}^{\mathbf{2}}=\mathbf{0 . 0 5}$} \\
\hline & $S E$-opt & $D$-opt & $E$-opt & Uniform \\
\hline \hline$\hat{K}_{\text {avg }}$ & 17.5009 & 17.4979 & 17.5021 & 17.5059 \\
\hline$\hat{K}_{\mathrm{SD}}$ & $1.022 \times 10^{-1}$ & $1.027 \times 10^{-1}$ & $8.133 \times 10^{-2}$ & $9.990 \times 10^{-2}$ \\
\hline$S E(\hat{K})$ & $9.987 \times 10^{-2}$ & $1.014 \times 10^{-1}$ & $7.906 \times 10^{-2}$ & $9.540 \times 10^{-2}$ \\
\hline$\hat{r}_{\text {avg }}$ & 0.7002 & 0.7005 & 0.7028 & 0.7007 \\
\hline$\hat{r}_{\mathrm{SD}}$ & $1.895 \times 10^{-2}$ & $2.091 \times 10^{-2}$ & $5.290 \times 10^{-2}$ & $2.748 \times 10^{-2}$ \\
\hline$S E(\hat{r})$ & $1.603 \times 10^{-2}$ & $1.716 \times 10^{-2}$ & $4.312 \times 10^{-2}$ & $2.248 \times 10^{-2}$ \\
\hline$\hat{x}_{0, \text { avg }}$ & 0.1007 & 0.1009 & 0.1044 & 0.1017 \\
\hline$\hat{x}_{0, \mathrm{SD}}$ & $1.334 \times 10^{-2}$ & $1.607 \times 10^{-2}$ & $3.612 \times 10^{-2}$ & $2.025 \times 10^{-2}$ \\
\hline$S E\left(\hat{x}_{0}\right)$ & $9.608 \times 10^{-3}$ & $1.110 \times 10^{-2}$ & $2.235 \times 10^{-2}$ & $1.385 \times 10^{-2}$ \\
\hline
\end{tabular}

Table 3: Average estimates $\hat{\theta}_{\text {avg }}$ and corresponding standard deviations $\hat{\theta}_{\mathrm{SD}}$ from $M=1000$ Monte Carlo trials as well as asymptotic standard errors $S E(\hat{\theta})$.

\begin{tabular}{|c||c|c|c|c|}
\hline \multicolumn{5}{|c|}{$\boldsymbol{N}=\mathbf{1 0}, \boldsymbol{\sigma}_{\mathbf{0}}^{\mathbf{2}}=\mathbf{0 . 1}$} \\
\hline & $S E$-opt & $D$-opt & $E$-opt & Uniform \\
\hline \hline$\hat{K}_{\text {avg }}$ & 17.5037 & 17.4942 & 17.4912 & 17.5067 \\
\hline$\hat{K}_{\text {SD }}$ & $1.390 \times 10^{-1}$ & $1.449 \times 10^{-1}$ & $1.069 \times 10^{-1}$ & $1.369 \times 10^{-1}$ \\
\hline$S E(\hat{K})$ & $1.412 \times 10^{-1}$ & $1.4339 \times 10^{-1}$ & $1.118 \times 10^{-1}$ & $1.349 \times 10^{-1}$ \\
\hline$\hat{r}_{\text {avg }}$ & 0.7004 & 0.7008 & 0.7079 & 0.7015 \\
\hline$\hat{r}_{\mathrm{SD}}$ & $2.756 \times 10^{-2}$ & $3.140 \times 10^{-2}$ & $7.641 \times 10^{-2}$ & $3.882 \times 10^{-2}$ \\
\hline$S E(\hat{r})$ & $2.266 \times 10^{-2}$ & $2.426 \times 10^{-2}$ & $6.098 \times 10^{-2}$ & $3.179 \times 10^{-2}$ \\
\hline$\hat{x}_{0, \text { avg }}$ & 0.1015 & 0.1023 & 0.1072 & 0.1028 \\
\hline$\hat{x}_{0, \mathrm{SD}}$ & $1.926 \times 10^{-2}$ & $2.441 \times 10^{-2}$ & $5.158 \times 10^{-2}$ & $2.832 \times 10^{-2}$ \\
\hline$S E\left(\hat{x}_{0}\right)$ & $1.359 \times 10^{-2}$ & $1.570 \times 10^{-2}$ & $3.161 \times 10^{-2}$ & $1.959 \times 10^{-2}$ \\
\hline
\end{tabular}

Table 4: Percent of confidence ellipsoids which contain the true parameter values $(\mathrm{N}=10)$.

\begin{tabular}{|c||c|c|c|}
\hline & $\sigma_{0}^{2}=0.01$ & $\sigma_{0}^{2}=0.05$ & $\sigma_{0}^{2}=0.10$ \\
\hline \hline$S E$-opt & $94.8 \%(S E=0.70 \%)$ & $94.5 \%(S E=0.72 \%)$ & $92.2 \%(S E=0.85 \%)$ \\
\hline$D$-opt & $94.7 \%(S E=0.71 \%)$ & $93.3 \%(S E=0.79 \%)$ & $88.3 \%(S E=1.02 \%)$ \\
\hline$E$-opt & $91.2 \%(S E=0.90 \%)$ & $82.7 \%(S E=1.20 \%)$ & $76.7 \%(S E=1.34 \%)$ \\
\hline Uniform & $94.4 \%(S E=0.73 \%)$ & $89.7 \%(S E=0.96 \%)$ & $89.0 \%(S E=0.99 \%)$ \\
\hline
\end{tabular}

\subsection{Discussion of logistic Monte Carlo results}

A general observation from these results is that as the measurement noise level decreases, variance in the parameters $\left(\hat{\theta}_{\mathrm{SD}}\right)$ decreases, and parameter estimates approach the true parameter values. 


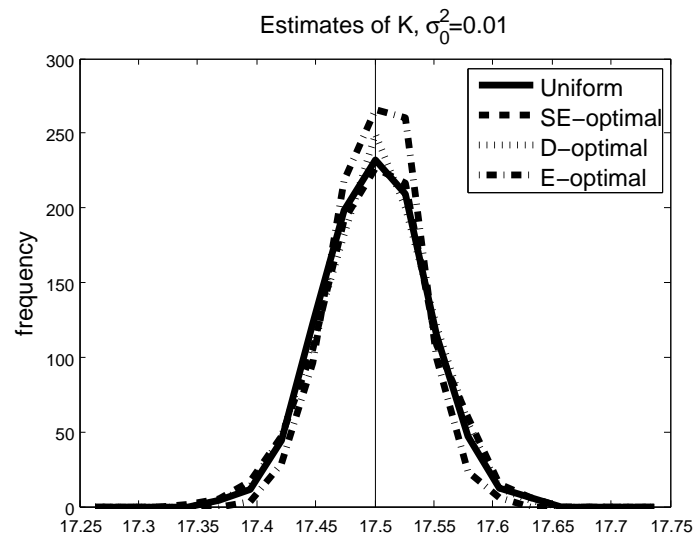

(a)

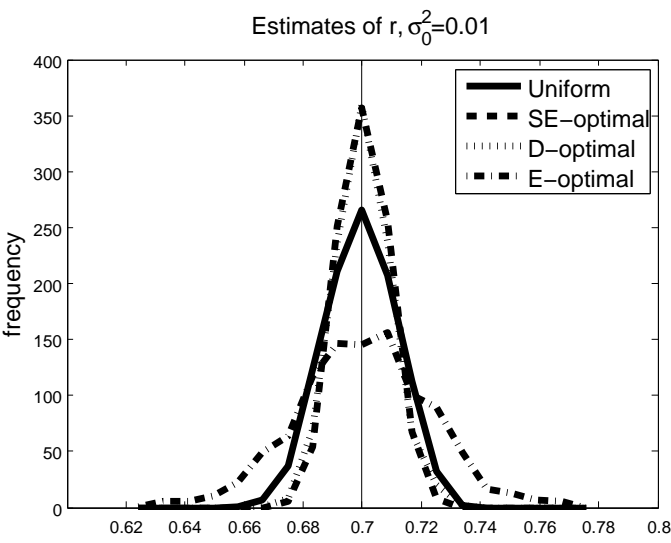

(b)

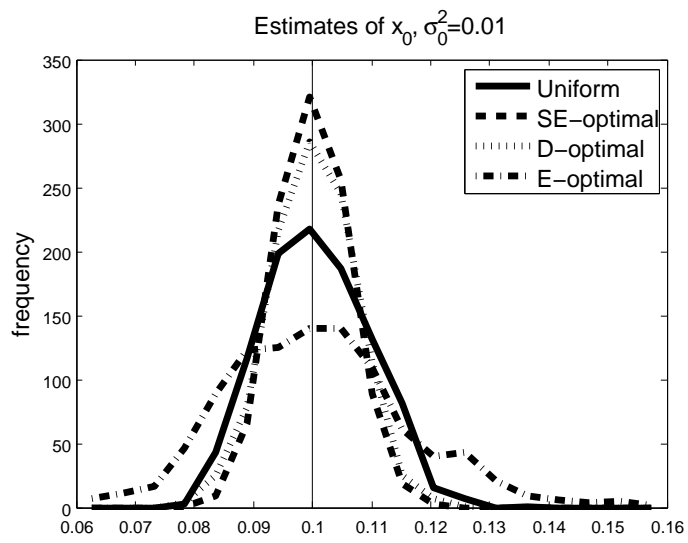

(c)

Figure 3: Histograms of parameter estimates ( $\hat{K}$ in panel (a), $\hat{r}$ in panel (b), $\hat{x}_{0}$ in panel (c)) resulting from Monte Carlo simulation with $M=1000$. Different histograms within each subfigure represent results from different optimal design methods as well as from the uniform mesh. Simulated data was generated with $N=10$ and $1 \%$ noise $\left(\sigma_{0}^{2}=0.01\right)$.

Comparing the standard deviation of the parameter estimates $\left(\hat{\theta}_{\mathrm{SD}}\right)$ to the asymptotic standard errors $(S E(\hat{\theta}))$ we find that they are always on the same order of magnitude, and often the asymptotic standard errors are slightly smaller (more optimistic).

In all cases the average parameter estimates were very close to the true values. Comparing the optimal design methods based on the standard deviations of the parameter estimates, we find different results based on the parameter. For parameter $K, E$-optimal had the smallest standard deviation in every case, followed by the uniform distribution. For parameters $r$ and $x_{0}, S E$-optimal always had the smallest standard deviation, often followed by $D$-optimal. Visually, $E$-optimal distributions appear right-skewed for parameters $r$ and $x_{0}$ for $\sigma_{0}^{2}=0.10$, and $N=10$ and $N=15$ (see Figs. 5 and 8). Overall, these results seem to be in agreement with the comparative performances of the optimal 


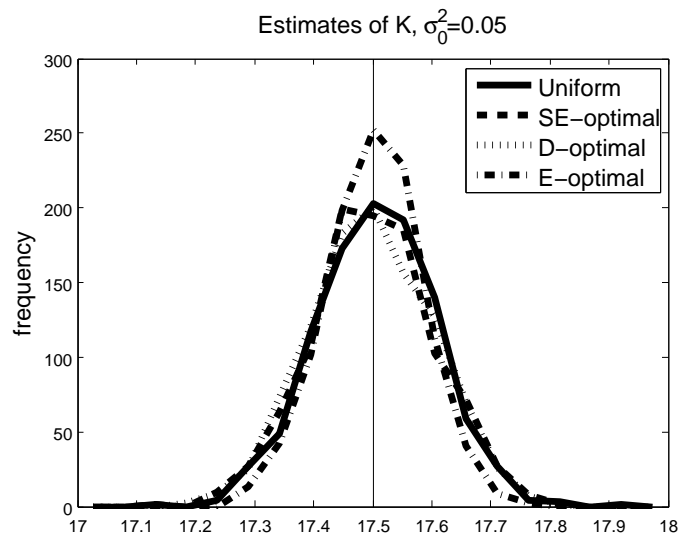

(a)

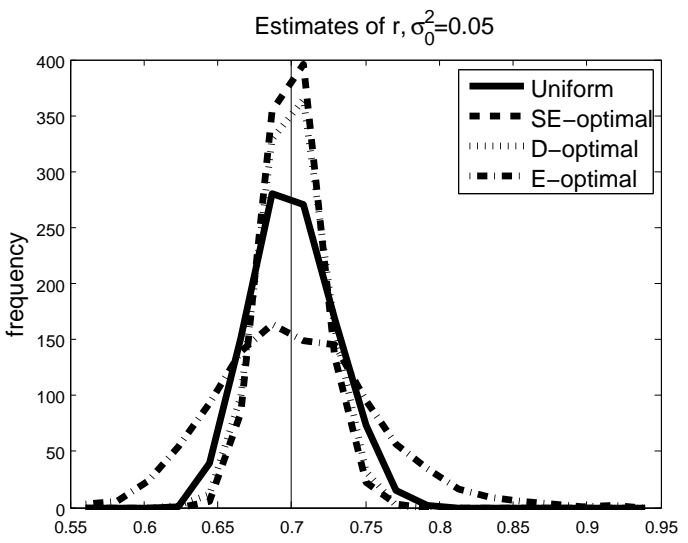

(b)

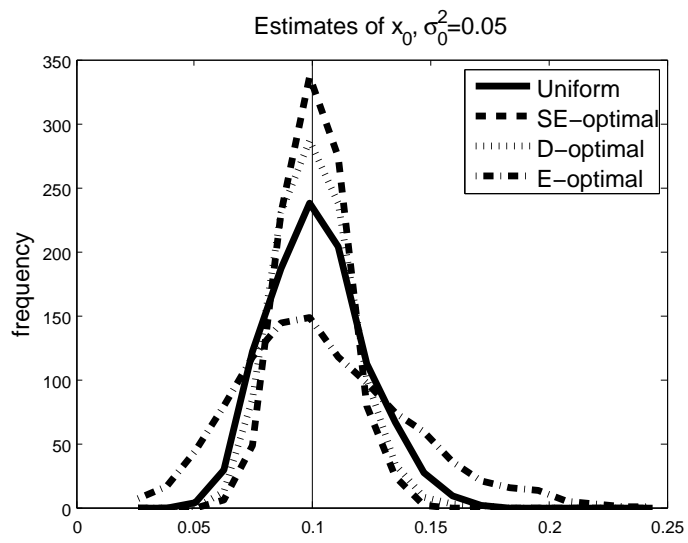

(c)

Figure 4: Histograms of parameter estimates ( $\hat{K}$ in panel (a), $\hat{r}$ in panel (b), $\hat{x}_{0}$ in panel (c)) resulting from Monte Carlo simulation with $M=1000$. Different histograms within each subfigure represent results from different optimal design methods as well as from the uniform mesh. Simulated data was generated with $N=10$ and $5 \%$ noise $\left(\sigma_{0}^{2}=0.05\right)$.

design methods presented in [8] where we used for comparison only asymptotic standard errors based on only one realization of the observation procedure.

Examining the proportion of $95 \%$ confidence ellipsoids that contain the true parameter value (Tables 4 and 8) we find that often the proportion falls short of the expected 0.95 , especially for increased values of $\sigma_{0}^{2}$. Comparing the optimal design methods based on which has the best coverage of the true parameter value, we find that $S E$-optimal is the best, followed by $D$-optimal or the uniform mesh, with $E$-optimal being the least desirable design method for this logistic example. 


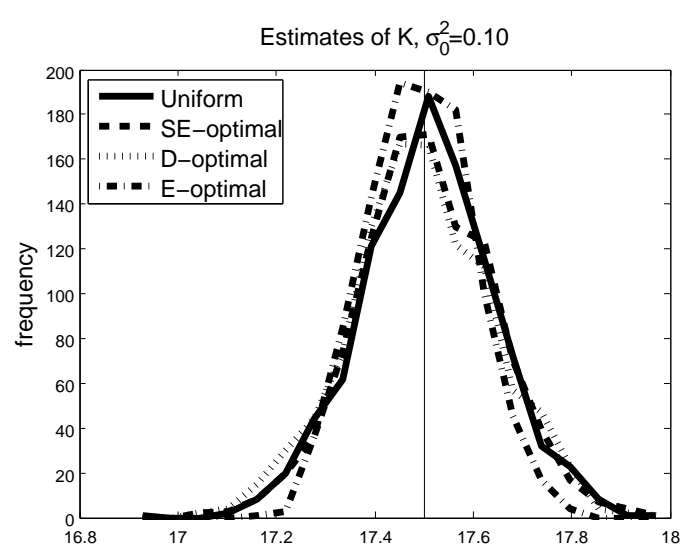

(a)

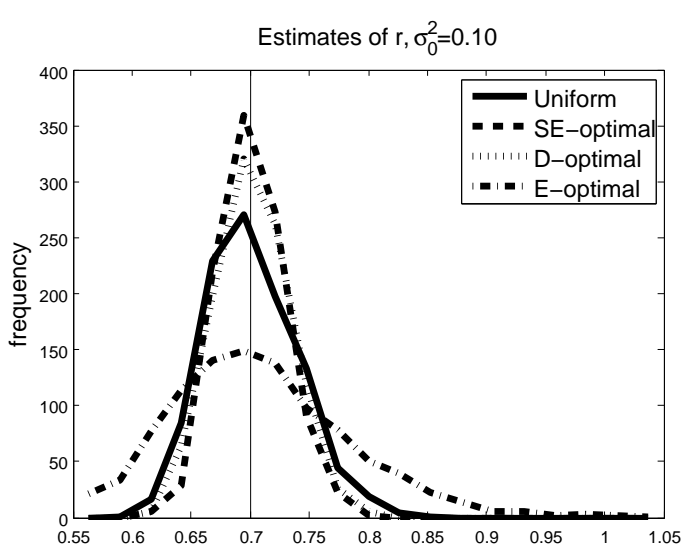

(b)

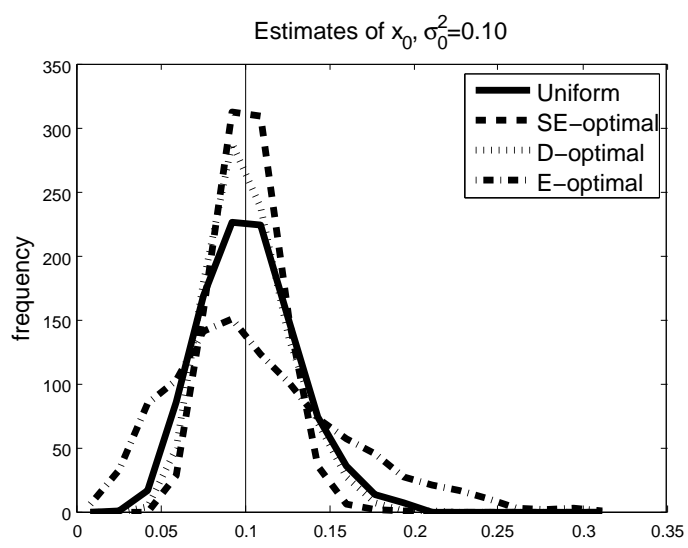

(c)

Figure 5: Histograms of parameter estimates ( $\hat{K}$ in panel (a), $\hat{r}$ in panel (b), $\hat{x}_{0}$ in panel (c)) resulting from Monte Carlo simulation with $M=1000$. Different histograms within each subfigure represent results from different optimal design methods as well as from the uniform mesh. Simulated data was generated with $N=10$ and $10 \%$ noise $\left(\sigma_{0}^{2}=0.10\right)$. 
Table 5: Average estimates $\hat{\theta}_{\mathrm{avg}}$ and corresponding standard deviations $\hat{\theta}_{\mathrm{SD}}$ from $M=1000$ Monte Carlo trials as well as asymptotic standard errors $S E(\hat{\theta})$.

\begin{tabular}{|c||c|c|c|c|}
\hline \multicolumn{5}{|c|}{$\boldsymbol{N}=\mathbf{1 5}, \boldsymbol{\sigma}_{\mathbf{0}}^{\mathbf{2}}=\mathbf{0 . 0 1}$} \\
\hline & $S E$-opt & $D$-opt & $E$-opt & Uniform \\
\hline \hline$\hat{K}_{\text {avg }}$ & 17.5001 & 17.4990 & 17.4980 & 17.5000 \\
\hline$\hat{K}_{\mathrm{SD}}$ & $3.747 \times 10^{-2}$ & $3.544 \times 10^{-2}$ & $2.914 \times 10^{-2}$ & $3.534 \times 10^{-2}$ \\
\hline$S E(\hat{K})$ & $3.774 \times 10^{-2}$ & $3.612 \times 10^{-2}$ & $3.015 \times 10^{-2}$ & $3.537 \times 10^{-2}$ \\
\hline$\hat{r}_{\text {avg }}$ & 0.7002 & 0.7000 & 0.7010 & 0.7001 \\
\hline$\hat{r}_{\mathrm{SD}}$ & $6.740 \times 10^{-3}$ & $8.543 \times 10^{-3}$ & $1.946 \times 10^{-2}$ & $9.622 \times 10^{-3}$ \\
\hline$S E(\hat{r})$ & $5.547 \times 10^{-3}$ & $6.862 \times 10^{-3}$ & $1.713 \times 10^{-2}$ & $8.088 \times 10^{-3}$ \\
\hline$\hat{x}_{0, \mathrm{avg}}$ & 0.0999 & 0.1002 & 0.1001 & 0.1002 \\
\hline$\hat{x}_{0, \mathrm{SD}}$ & $4.895 \times 10^{-3}$ & $6.309 \times 10^{-3}$ & $1.197 \times 10^{-2}$ & $6.993 \times 10^{-3}$ \\
\hline$S E\left(\hat{x}_{0}\right)$ & $3.524 \times 10^{-3}$ & $4.185 \times 10^{-3}$ & $7.560 \times 10^{-3}$ & $4.989 \times 10^{-3}$ \\
\hline
\end{tabular}

Table 6: Average estimates $\hat{\theta}_{\text {avg }}$ and corresponding standard deviations $\hat{\theta}_{\mathrm{SD}}$ from $M=1000$ Monte Carlo trials as well as asymptotic standard errors $S E(\hat{\theta})$.

\begin{tabular}{|c||c|c|c|c|}
\hline \multicolumn{5}{|c|}{$\boldsymbol{N}=\mathbf{1 5}, \boldsymbol{\sigma}_{\mathbf{0}}^{\mathbf{2}}=\mathbf{0 . 0 5}$} \\
\hline & $S E$-opt & $D$-opt & $E$-opt & Uniform \\
\hline \hline$\hat{K}_{\text {avg }}$ & 17.4980 & 17.5029 & 17.4994 & 17.5002 \\
\hline$\hat{K}_{\text {SD }}$ & $8.612 \times 10^{-2}$ & $7.832 \times 10^{-2}$ & $6.850 \times 10^{-2}$ & $7.883 \times 10^{-2}$ \\
\hline$S E(\hat{K})$ & $8.438 \times 10^{-2}$ & $8.077 \times 10^{-2}$ & $6.742 \times 10^{-2}$ & $7.910 \times 10^{-2}$ \\
\hline$\hat{r}_{\text {avg }}$ & 0.7009 & 0.7005 & 0.7017 & 0.7008 \\
\hline$\hat{r}_{\text {SD }}$ & $1.529 \times 10^{-2}$ & $1.966 \times 10^{-2}$ & $4.524 \times 10^{-2}$ & $2.250 \times 10^{-2}$ \\
\hline$S E(\hat{r})$ & $1.240 \times 10^{-2}$ & $1.534 \times 10^{-2}$ & $3.831 \times 10^{-2}$ & $1.809 \times 10^{-2}$ \\
\hline$\hat{x}_{0, \text { avg }}$ & 0.1000 & 0.1006 & 0.1028 & 0.1007 \\
\hline$\hat{x}_{0, \mathrm{SD}}$ & $1.120 \times 10^{-2}$ & $1.453 \times 10^{-2}$ & $2.757 \times 10^{-2}$ & $1.647 \times 10^{-2}$ \\
\hline$S E\left(\hat{x}_{0}\right)$ & $7.880 \times 10^{-3}$ & $9.357 \times 10^{-3}$ & $1.690 \times 10^{-2}$ & $1.116 \times 10^{-2}$ \\
\hline
\end{tabular}


Table 7: Average estimates $\hat{\theta}_{\text {avg }}$ and corresponding standard deviations $\hat{\theta}_{\mathrm{SD}}$ from $M=1000$ Monte Carlo trials as well as asymptotic standard errors $S E(\hat{\theta})$.

\begin{tabular}{|c||c|c|c|c|}
\hline \multicolumn{5}{|c|}{$\boldsymbol{N}=\mathbf{1 5}, \boldsymbol{\sigma}_{\mathbf{0}}^{\mathbf{2}}=\mathbf{0 . 1}$} \\
\hline & $S E$-opt & $D$-opt & $E$-opt & Uniform \\
\hline \hline$\hat{K}_{\text {avg }}$ & 17.4987 & 17.5014 & 17.5014 & 17.5071 \\
\hline$\hat{K}_{\mathrm{SD}}$ & $1.192 \times 10^{-1}$ & $1.135 \times 10^{-1}$ & $9.545 \times 10^{-2}$ & $1.151 \times 10^{-1}$ \\
\hline$S E(\hat{K})$ & $1.193 \times 10^{-1}$ & $1.142 \times 10^{-1}$ & $9.535 \times 10^{-2}$ & $1.119 \times 10^{-1}$ \\
\hline$\hat{r}_{\text {avg }}$ & 0.7011 & 0.7017 & 0.7046 & 0.7010 \\
\hline$\hat{r}_{\mathrm{SD}}$ & $2.070 \times 10^{-2}$ & $2.703 \times 10^{-2}$ & $6.565 \times 10^{-2}$ & $3.132 \times 10^{-2}$ \\
\hline$S E(\hat{r})$ & $1.754 \times 10^{-2}$ & $2.170 \times 10^{-2}$ & $5.417 \times 10^{-2}$ & $2.558 \times 10^{-2}$ \\
\hline$\hat{x}_{0, \text { avg }}$ & 0.1007 & 0.1009 & 0.1043 & 0.1018 \\
\hline$\hat{x}_{0, \mathrm{SD}}$ & $1.530 \times 10^{-2}$ & $2.026 \times 10^{-2}$ & $4.032 \times 10^{-2}$ & $2.317 \times 10^{-2}$ \\
\hline$S E\left(\hat{x}_{0}\right)$ & $1.114 \times 10^{-2}$ & $1.323 \times 10^{-2}$ & $2.391 \times 10^{-2}$ & $1.578 \times 10^{-2}$ \\
\hline
\end{tabular}

Table 8: Percent of confidence ellipsoids which contain the true parameter values $(\mathrm{N}=15)$.

\begin{tabular}{|c||c|c|c|}
\hline & $\sigma_{0}^{2}=0.01$ & $\sigma_{0}^{2}=0.05$ & $\sigma_{0}^{2}=0.10$ \\
\hline \hline$S E$-opt & $95.7 \%(S E=0.64 \%)$ & $94.0 \%(S E=0.75 \%)$ & $93.5 \%(S E=0.78 \%)$ \\
\hline$D$-opt & $95.1 \%(S E=0.68 \%)$ & $92.3 \%(S E=0.84 \%)$ & $89.2 \%(S E=0.98 \%)$ \\
\hline$E$-opt & $93.9 \%(S E=0.76 \%)$ & $87.8 \%(S E=1.04 \%)$ & $82.5 \%(S E=1.20 \%)$ \\
\hline Uniform & $94.0 \%(S E=0.75 \%)$ & $91.2 \%,(S E=0.90 \%)$ & $89.7 \%(S E=0.96 \%)$ \\
\hline
\end{tabular}




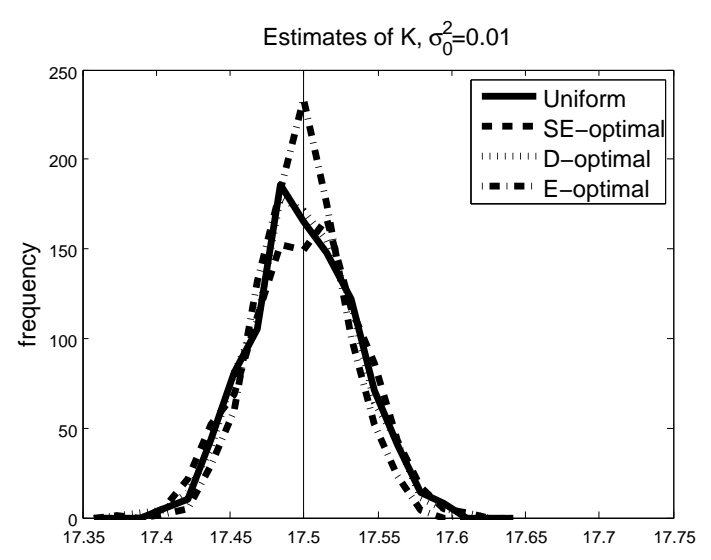

(a)

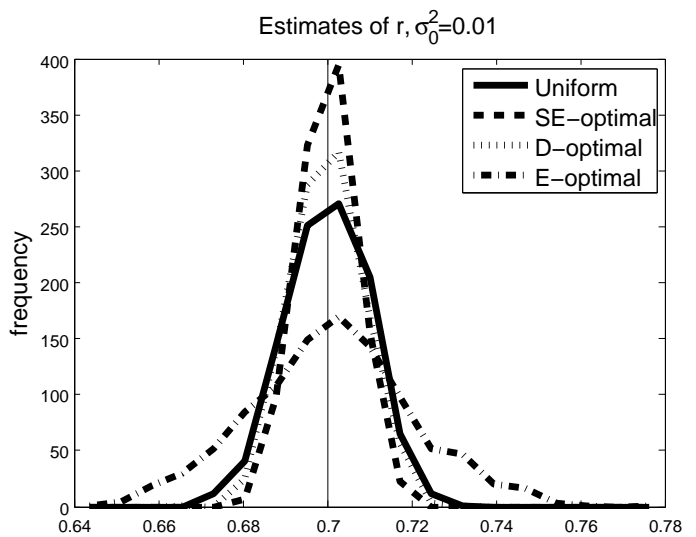

(b)

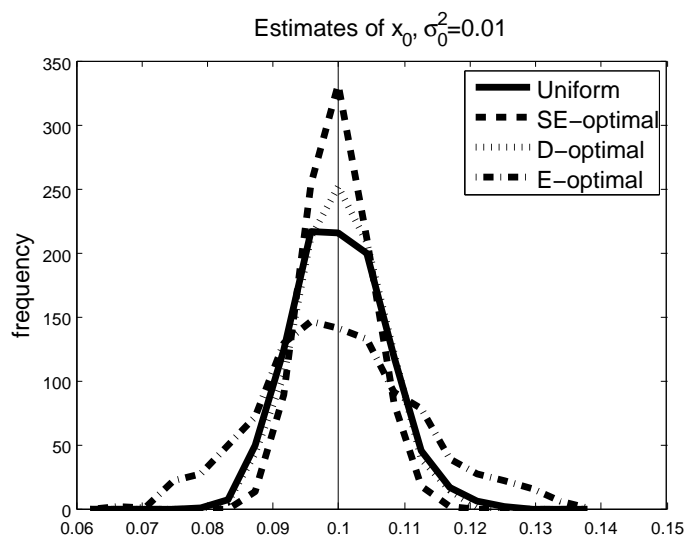

(c)

Figure 6: Histograms of parameter estimates ( $\hat{K}$ in panel (a), $\hat{r}$ in panel (b), $\hat{x}_{0}$ in panel (c)) resulting from Monte Carlo simulation with $M=1000$. Different histograms within each subfigure represent results from different optimal design methods as well as from the uniform mesh. Simulated data was generated with $N=15$ and $1 \%$ noise $\left(\sigma_{0}^{2}=0.01\right)$. 


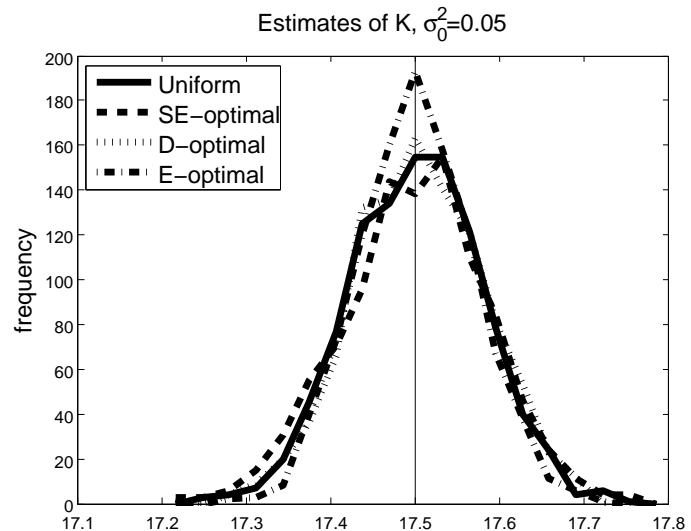

(a)

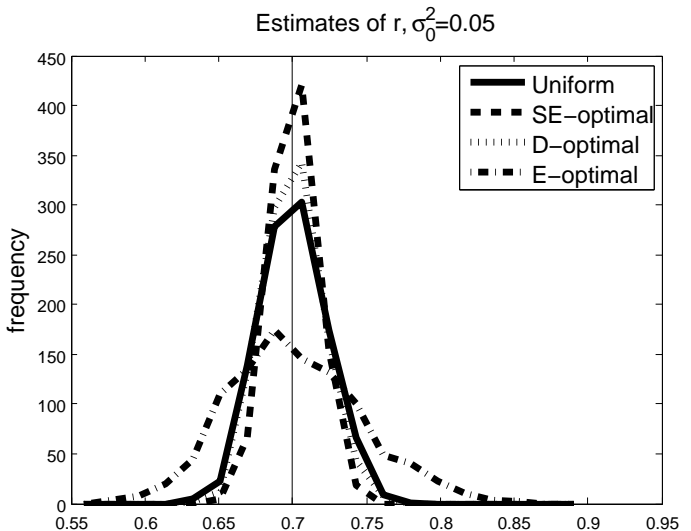

(b)

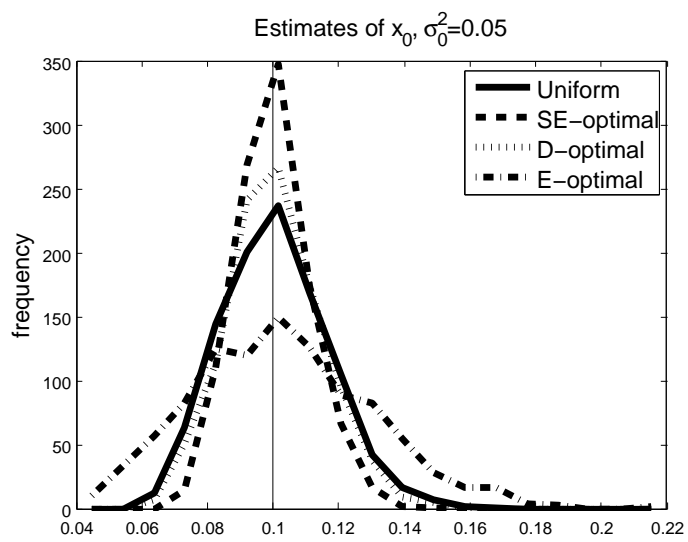

(c)

Figure 7: Histograms of parameter estimates ( $\hat{K}$ in panel (a), $\hat{r}$ in panel (b), $\hat{x}_{0}$ in panel (c)) resulting from Monte Carlo simulation with $M=1000$. Different histograms within each subfigure represent results from different optimal design methods as well as from the uniform mesh. Simulated data was generated with $N=15$ and $5 \%$ noise $\left(\sigma_{0}^{2}=0.05\right)$. 


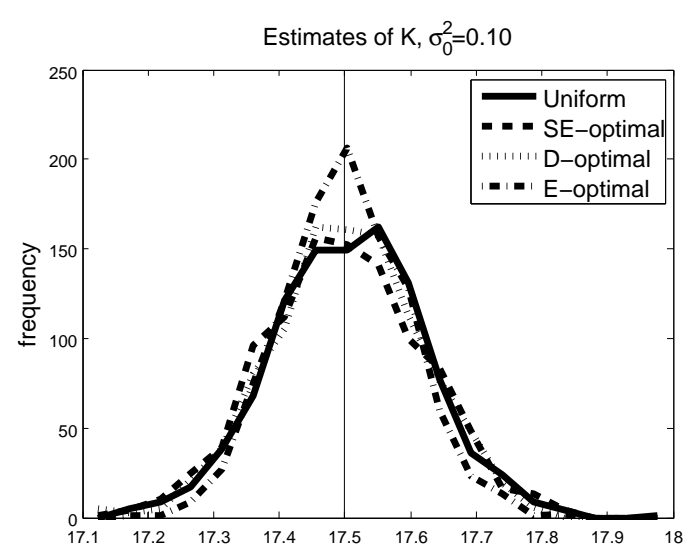

(a)

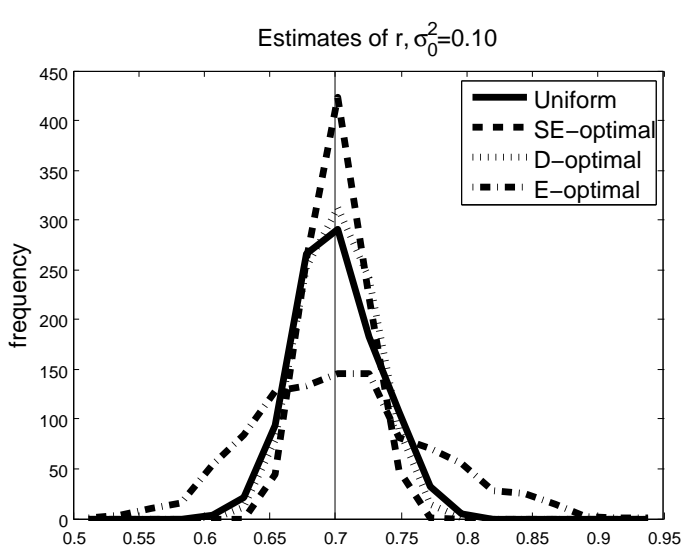

(b)

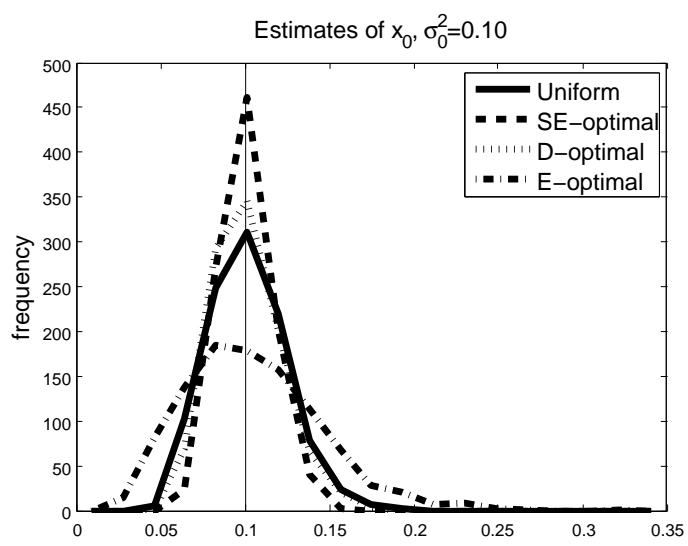

(c)

Figure 8: Histograms of parameter estimates ( $\hat{K}$ in panel (a), $\hat{r}$ in panel (b), $\hat{x}_{0}$ in panel (c)) resulting from Monte Carlo simulation with $M=1000$. Different histograms within each subfigure represent results from different optimal design methods as well as from the uniform mesh. Simulated data was generated with $N=15$ and $10 \%$ noise $\left(\sigma_{0}^{2}=0.10\right)$. 


\section{$5 \quad$ Harmonic oscillator results}

\subsection{The harmonic oscillator}

Harmonic oscillators are ubiquitous in science, being found in a number of applications ranging from biology (Lorentz electromagnetic polarization laws in tissue), electronic circuits and mechanical devices. The model for the harmonic oscillator can be easily derived (see [10]) using Hooke's Law and Newton's Second Law of Motion and is given with out external forcing by

$$
m \ddot{x}+c \dot{x}+k x=0, \quad \dot{x}(0)=x_{1}, \quad x(0)=x_{2} .
$$

Here, $m$ is mass, $c$ is the damping coefficient, and $k$ is the spring constant. The parameters $m, c$ and $k$ are not independent for the unforced oscillator. Dividing through by $m$, and defining $C=c / m$ and $K=k / m$, we can reduce the number of parameters and obtain

$$
\ddot{x}+C \dot{x}+K x=0, \quad \dot{x}(0)=x_{1}, \quad x(0)=x_{2} .
$$

The analytical solution of the initial value problem (5.1) is given by

$$
x(t)=x(t, \theta)=e^{-a t}\left(x_{2} \cos b t+\frac{x_{1}+a x_{2}}{b} \sin b t\right) \text { for } 0 \leq t \leq T,
$$

where $a=\frac{1}{2} C, b=\sqrt{K-\frac{1}{4} C^{2}}$. For the following investigations we fixed the initial values in problem (5.1) to the values $x_{1}=-1, x_{2}=0.5$. The parameter vector considered is $\theta=(C, K)^{\top}$.

For our Monte Carlo analysis we choose the nominal parameter vector

$$
\theta_{0}=\left(C_{\text {nom }}, K_{\text {nom }}\right)^{\top}=(0.1,0.2)^{\top}
$$

and take displacement $x(t, \theta)$ as the model output so that

$$
f(t, \theta)=x(t, \theta), \quad t \geq 0 .
$$

\subsection{Monte Carlo analysis for the harmonic oscillator model}

Monte Carlo analysis was conducted using the optimal design points computed with $N=15, T=$ 14.14 or $T=28.28$, and the four different implementations $(\mathrm{C} 1)-(\mathrm{C} 4)$ of the constraints (see Figures 9 and 10). For each optimal mesh or the uniform mesh, the Monte Carlo analysis was repeated three times for different constant variance $\left(\sigma^{2}(t)=\sigma_{0}^{2}\right.$ for all $\left.t\right)$ noise levels $\sigma_{0}^{2}=(0.01,0.05,0.10)$. Figures $11-18$ contain histogram plots of the parameter estimates $(\hat{C}, \hat{K})$. Each figure represents results from a specific constraint implementation and a value of $T$. Each subfigure represents one of the parameters and one value of $\sigma_{0}^{2}$. Within a subfigure are histograms corresponding to each optimal design method ( $S E$-optimal, $D$-optimal, and $E$-optimal) and the uniform mesh. 


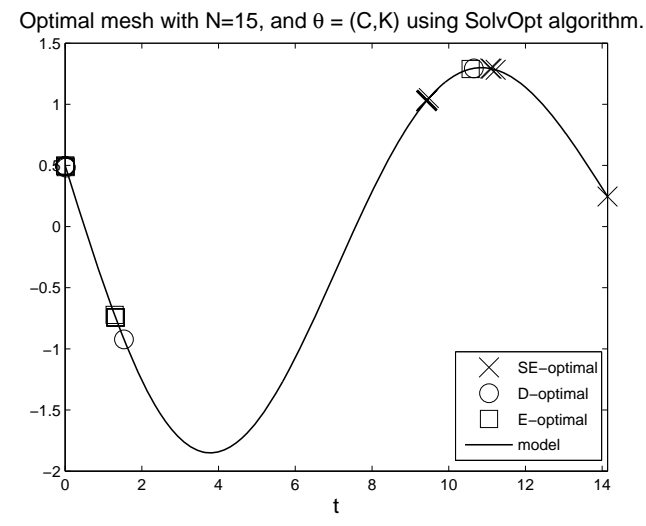

Optimal mesh with $\mathrm{N}=15$, and $\theta=(\mathrm{C}, \mathrm{K})$ using SolvOpt algorithm.

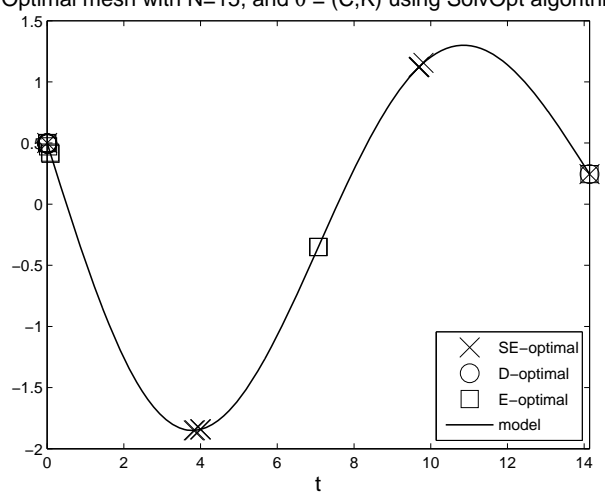

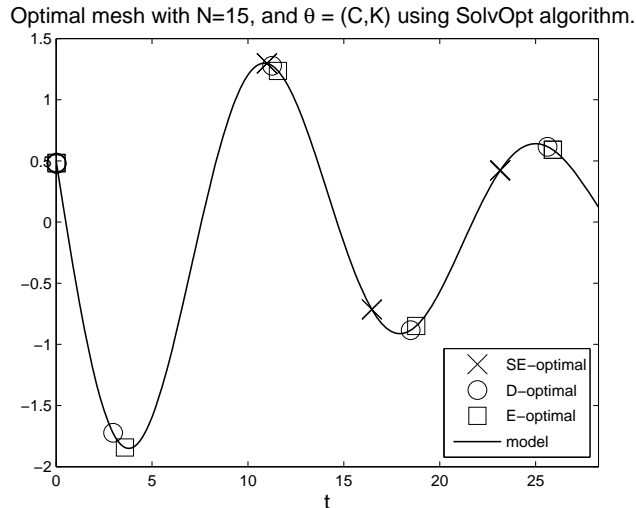

Optimal mesh with $\mathrm{N}=15$, and $\theta=(\mathrm{C}, \mathrm{K})$ using SolvOpt algorithm

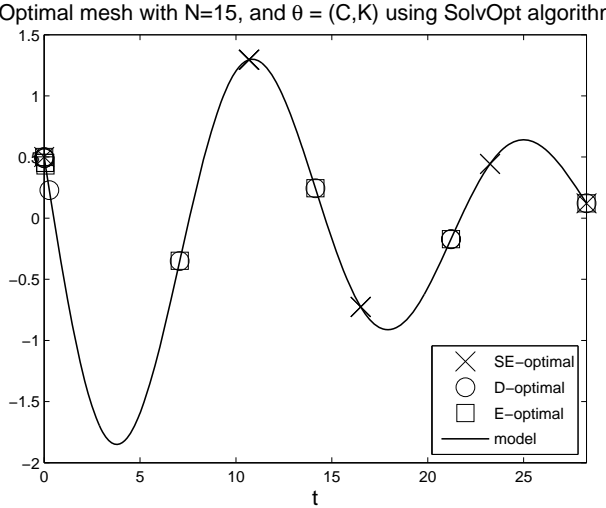

Figure 9: Optimal sampling times according to the design criteria for the harmonic oscillator. Optimal sampling times were obtained using SolvOpt with $\mathrm{N}=15$, constraint implementation (C1) (upper panels) respectively $(\mathrm{C} 2)$ (lower panels) and $T=14.14$ (left panels) respectively $T=28.28$ (right panels). 


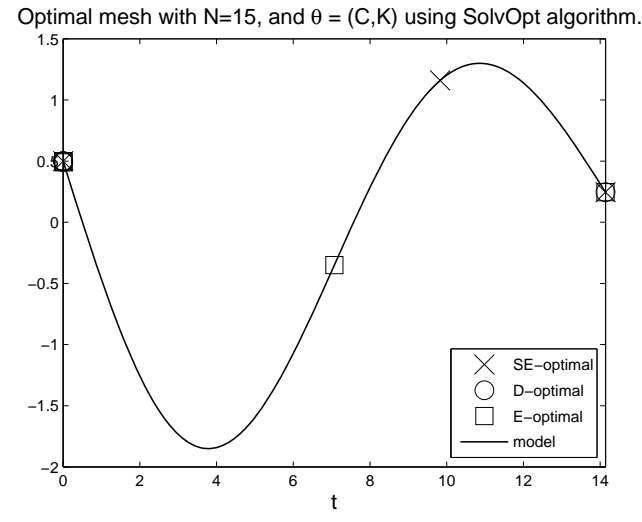

Optimal mesh with $\mathrm{N}=15$, and $\theta=(\mathrm{C}, \mathrm{K})$ using SolvOpt algorithm.

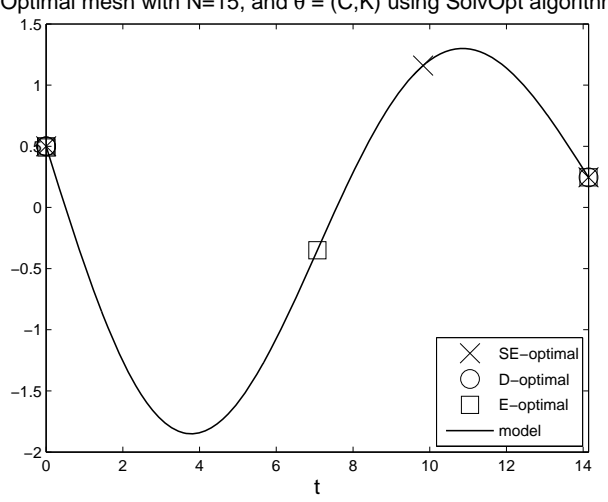

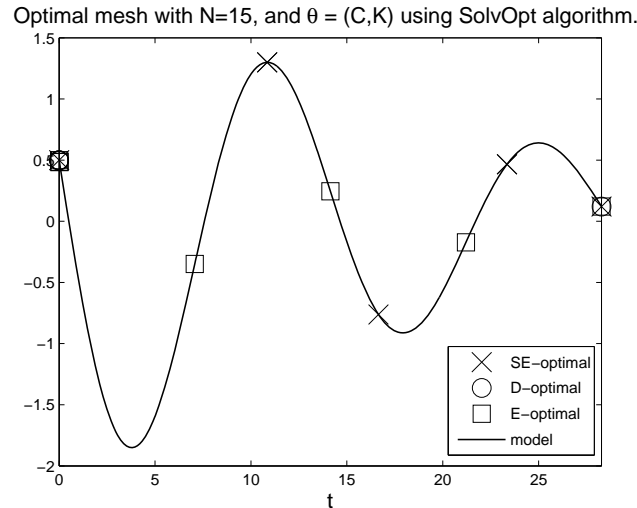

Optimal mesh with $\mathrm{N}=15$, and $\theta=(\mathrm{C}, \mathrm{K})$ using SolvOpt algorithm.

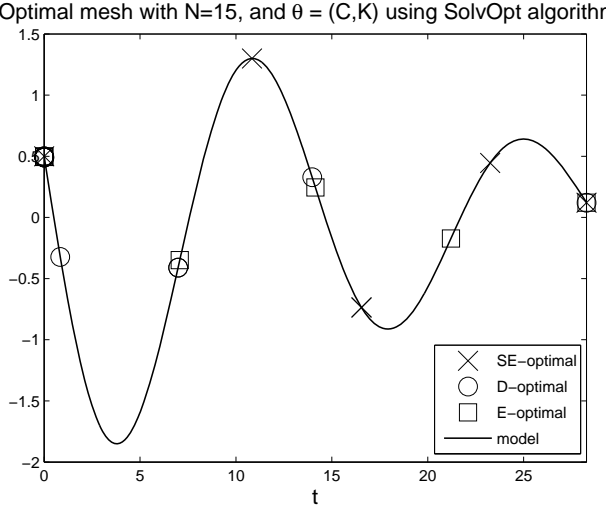

Figure 10: Optimal sampling times according to the design criteria for the harmonic oscillator. Optimal sampling times were obtained using SolvOpt with $\mathrm{N}=15$, constraint implementation (C3) (upper panels) respectively (C4) (lower panels) and $T=14.14$ (left panels) respectively $T=28.28$ (right panels). 
Tables $9-11,14-16,19-21$, and $24-26$ contain the average estimates and standard deviations from the Monte Carlo trials for the four constraints respectively, and both $T=14.14$ and $T=28.28$, as well as for the three different noise levels. In addition, the tables contain the asymptotic standard errors as computed in (2.7) using the true parameter values, for comparison with the standard deviations of the Monte Carlo parameter estimates. The percentage of $95 \%$ confidence ellipsoids which contain the true parameter values are given in Tables 12, 13, 17, 18, 22, 23, 27, and 28.

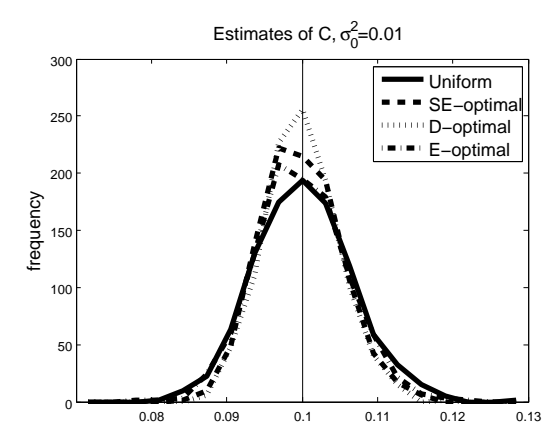

(a)

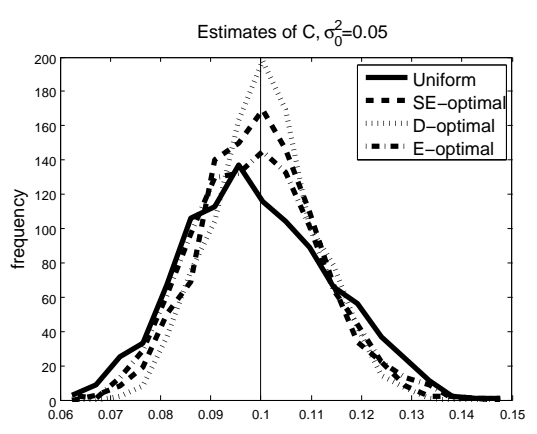

(c)

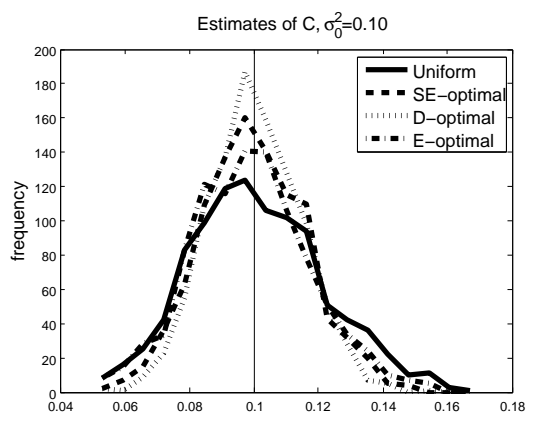

(e)

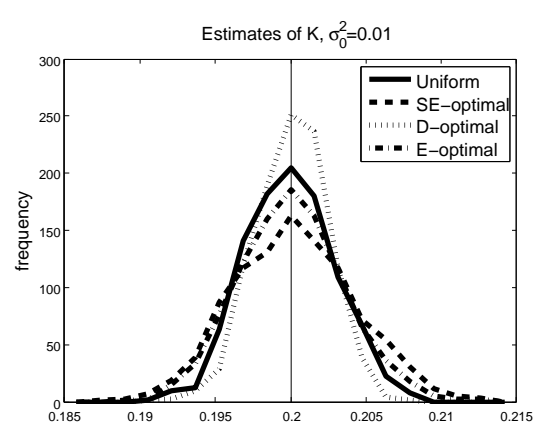

(b)

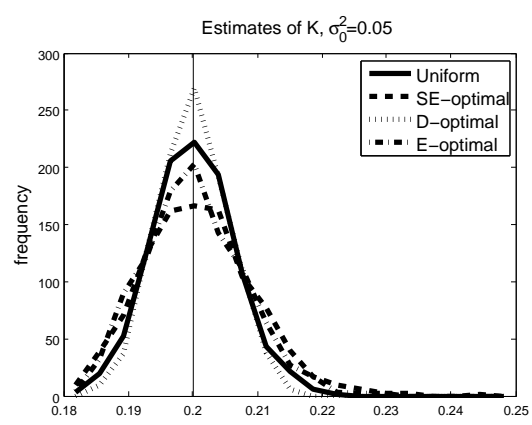

(d)

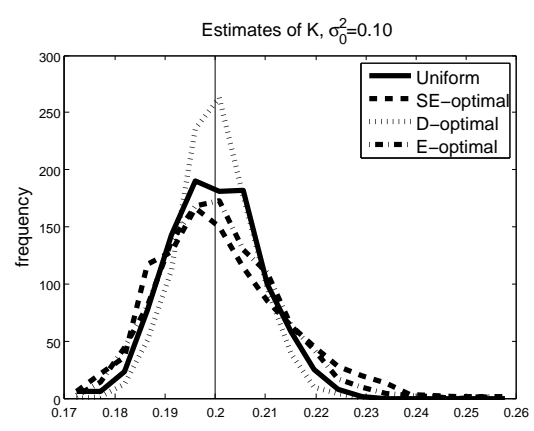

(f)

Figure 11: Histograms of parameter estimates $(\hat{C}$ in panels (a),(c),(e), and $\hat{K}$ in panels (b),(d),(f)) resulting from Monte Carlo simulation. Simulated data was generated with $\sigma_{0}^{2}=0.01$ (top row), $\sigma_{0}^{2}=0.05$ (middle row), and $\sigma_{0}^{2}=0.10$ (bottom row) $(T=14.14$ and constraint implementation (C1)). 
Table 9: Average estimates $\hat{\theta}_{\text {avg }}$ with corresponding standard deviations $\hat{\theta}_{\mathrm{SD}}$ from $M=1000$ Monte Carlo trials as well as asymptotic standard errors $S E(\hat{\theta})$. Estimation with constraint implementation (C1).

\begin{tabular}{|c|c|c|c|c|}
\hline \multicolumn{5}{|c|}{$N=15, \sigma_{0}^{2}=0.01$} \\
\hline \multicolumn{5}{|c|}{$T=14.14$} \\
\hline & $S E$-opt & $D$-opt & $E$-opt & Uniform \\
\hline$\hat{C}_{\text {avg }}$ & 0.0998 & 0.0999 & 0.0999 & 0.1002 \\
\hline$\hat{C}_{\mathrm{SD}}$ & $5.197 \times 10^{-3}$ & $4.904 \times 10^{-3}$ & $5.928 \times 10^{-3}$ & $6.495 \times 10^{-3}$ \\
\hline$S E(\hat{C})$ & $5.004 \times 10^{-3}$ & $4.746 \times 10^{-3}$ & $5.818 \times 10^{-3}$ & $6.577 \times 10^{-3}$ \\
\hline$\hat{K}_{\text {avg }}$ & 0.2002 & 0.2001 & 0.2000 & 0.2000 \\
\hline$\hat{K}_{\mathrm{SD}}$ & $4.049 \times 10^{-3}$ & $2.360 \times 10^{-3}$ & $3.482 \times 10^{-3}$ & $2.977 \times 10^{-3}$ \\
\hline$S E(\hat{K})$ & $3.678 \times 10^{-3}$ & $2.378 \times 10^{-3}$ & $3.416 \times 10^{-3}$ & $2.895 \times 10^{-3}$ \\
\hline \multicolumn{5}{|c|}{$T=28.28$} \\
\hline$\hat{C}_{\text {avg }}$ & 0.1000 & 0.0999 & 0.0999 & 0.1000 \\
\hline$\hat{C}_{\mathrm{SD}}$ & $4.493 \times 10^{-3}$ & $4.319 \times 10^{-3}$ & $5.707 \times 10^{-3}$ & $5.123 \times 10^{-3}$ \\
\hline$S E(\hat{C})$ & $4.342 \times 10^{-3}$ & $4.284 \times 10^{-3}$ & $5.214 \times 10^{-3}$ & $5.127 \times 10^{-3}$ \\
\hline$\hat{K}_{\text {avg }}$ & 0.2002 & 0.2000 & 0.2002 & 0.2000 \\
\hline$\hat{K}_{\mathrm{SD}}$ & $3.238 \times 10^{-3}$ & $1.854 \times 10^{-3}$ & $3.981 \times 10^{-3}$ & $2.413 \times 10^{-3}$ \\
\hline$S E(\hat{K})$ & $3.021 \times 10^{-3}$ & $1.994 \times 10^{-3}$ & $3.412 \times 10^{-3}$ & $2.307 \times 10^{-3}$ \\
\hline
\end{tabular}

Table 10: Average estimates $\hat{\theta}_{\text {avg }}$ with corresponding standard deviations $\hat{\theta}_{\mathrm{SD}}$ from $M=1000$ Monte Carlo trials as well as asymptotic standard errors $S E(\hat{\theta})$. Estimation with constraint implementation (C1).

\begin{tabular}{|c||c|c|c|c|}
\hline \multicolumn{5}{|c|}{$\mathbf{T}=\mathbf{1 5}, \boldsymbol{\sigma}_{\mathbf{0}}^{\mathbf{2}}=\mathbf{0 . 0 5}$} \\
\hline \multicolumn{5}{|c|}{$\mathbf{1 4 . 1 4}$} \\
\hline & $S E$-opt & $D$-opt & $E$-opt & Uniform \\
\hline \hline$\hat{C}_{\text {avg }}$ & 0.0999 & 0.1008 & 0.0994 & 0.0994 \\
\hline$\hat{C}_{\text {SD }}$ & $1.145 \times 10^{-2}$ & $1.019 \times 10^{-2}$ & $1.286 \times 10^{-2}$ & $1.457 \times 10^{-2}$ \\
\hline$S E(\hat{C})$ & $1.119 \times 10^{-2}$ & $1.061 \times 10^{-2}$ & $1.301 \times 10^{-2}$ & $1.471 \times 10^{-2}$ \\
\hline$\hat{K}_{\text {avg }}$ & 0.2008 & 0.2000 & 0.2001 & 0.2001 \\
\hline$\hat{K}_{\text {SD }}$ & $8.782 \times 10^{-3}$ & $5.430 \times 10^{-3}$ & $7.761 \times 10^{-3}$ & $6.469 \times 10^{-3}$ \\
\hline$S E(\hat{K})$ & $8.224 \times 10^{-3}$ & $5.318 \times 10^{-3}$ & $7.640 \times 10^{-3}$ & $6.473 \times 10^{-3}$ \\
\hline \multicolumn{5}{|c|}{$\mathbf{T}=\mathbf{2 8 . 2 8}$} \\
\hline$\hat{C}_{\text {avg }}$ & 0.0996 & 0.0997 & 0.0988 & 0.1001 \\
\hline$\hat{C}_{\text {SD }}$ & $9.659 \times 10^{-3}$ & $9.588 \times 10^{-3}$ & $1.224 \times 10^{-2}$ & $1.183 \times 10^{-2}$ \\
\hline$S E(\hat{C})$ & $9.708 \times 10^{-3}$ & $9.579 \times 10^{-3}$ & $1.166 \times 10^{-2}$ & $1.146 \times 10^{-2}$ \\
\hline$\hat{K}_{\text {avg }}$ & 0.2004 & 0.2000 & 0.2013 & 0.2001 \\
\hline$\hat{K}_{\text {SD }}$ & $7.795 \times 10^{-3}$ & $4.295 \times 10^{-3}$ & $1.129 \times 10^{-2}$ & $5.114 \times 10^{-3}$ \\
\hline$S E(\hat{K})$ & $6.755 \times 10^{-3}$ & $4.459 \times 10^{-3}$ & $7.629 \times 10^{-3}$ & $5.159 \times 10^{-3}$ \\
\hline$S$
\end{tabular}


Table 11: Average estimates $\hat{\theta}_{\text {avg }}$ with corresponding standard deviations $\hat{\theta}_{\mathrm{SD}}$ from $M=1000$ Monte Carlo trials as well as asymptotic standard errors $S E(\hat{\theta})$. Estimation with constraint implementation (C1).

\begin{tabular}{|c||c|c|c|c|}
\hline \multicolumn{5}{|c|}{$\boldsymbol{N}=\mathbf{1 5}, \boldsymbol{\sigma}_{\mathbf{0}}^{\mathbf{2}}=\mathbf{0 . 1}$} \\
\hline \multicolumn{5}{|c|}{$\mathbf{1 4 . 1 4}$} \\
\hline & $S E$-opt & $D$-opt & $E$-opt & Uniform \\
\hline \hline$\hat{C}_{\text {avg }}$ & 0.0999 & 0.1003 & 0.0991 & 0.1008 \\
\hline$\hat{C}_{\mathrm{SD}}$ & $1.621 \times 10^{-2}$ & $1.445 \times 10^{-2}$ & $1.842 \times 10^{-2}$ & $2.107 \times 10^{-2}$ \\
\hline$S E(\hat{C})$ & $1.582 \times 10^{-2}$ & $1.501 \times 10^{-2}$ & $1.840 \times 10^{-2}$ & $2.080 \times 10^{-2}$ \\
\hline$\hat{K}_{\text {avg }}$ & 0.2008 & 0.2002 & 0.2007 & 0.2000 \\
\hline$\hat{K}_{\mathrm{SD}}$ & $1.286 \times 10^{-2}$ & $7.447 \times 10^{-3}$ & $1.131 \times 10^{-2}$ & $9.192 \times 10^{-3}$ \\
\hline$S E(\hat{K})$ & $1.163 \times 10^{-2}$ & $7.521 \times 10^{-3}$ & $1.080 \times 10^{-2}$ & $9.154 \times 10^{-3}$ \\
\hline \multicolumn{5}{|c|}{$\mathbf{T}=\mathbf{2 8 . 2 8}$} \\
\hline$\hat{C}_{\text {avg }}$ & 0.0991 & 0.0998 & 0.0978 & 0.0992 \\
\hline$\hat{C}_{\mathrm{SD}}$ & $1.386 \times 10^{-2}$ & $1.353 \times 10^{-2}$ & $1.714 \times 10^{-2}$ & $1.628 \times 10^{-2}$ \\
\hline$S E(\hat{C})$ & $1.373 \times 10^{-2}$ & $1.355 \times 10^{-2}$ & $1.649 \times 10^{-2}$ & $1.621 \times 10^{-2}$ \\
\hline$\hat{K}_{\text {avg }}$ & 0.2015 & 0.2003 & 0.2038 & 0.2001 \\
\hline$\hat{K}_{\mathrm{SD}}$ & $1.106 \times 10^{-2}$ & $6.602 \times 10^{-3}$ & $1.807 \times 10^{-2}$ & $7.350 \times 10^{-3}$ \\
\hline$S E(\hat{K})$ & $9.553 \times 10^{-3}$ & $6.305 \times 10^{-3}$ & $1.079 \times 10^{-2}$ & $7.295 \times 10^{-3}$ \\
\hline
\end{tabular}

Table 12: Percent of confidence ellipsoids which contain the true parameter values $(T=14.14$, constraint implementation $(\mathrm{C} 1))$.

\begin{tabular}{|c||c|c|c|}
\hline & $\sigma_{0}^{2}=0.01$ & $\sigma_{0}^{2}=0.05$ & $\sigma_{0}^{2}=0.10$ \\
\hline \hline$S E$-opt & $95.9 \%(S E=0.63 \%)$ & $94.7 \%(S E=0.71 \%)$ & $94.3 \%(S E=0.73 \%)$ \\
\hline$D$-opt & $95.2 \%(S E=0.68 \%)$ & $96.7 \%(S E=0.56 \%)$ & $95.9 \%(S E=0.63 \%)$ \\
\hline$E$-opt & $95.5 \%(S E=0.66 \%)$ & $94.4 \%(S E=0.73 \%)$ & $94.6 \%(S E=0.71 \%)$ \\
\hline Uniform & $94.6 \%(S E=0.71 \%)$ & $95.2 \%(S E=0.68 \%)$ & $95.4 \%(S E=0.66 \%)$ \\
\hline
\end{tabular}

Table 13: Percent of confidence ellipsoids which contain the true parameter values $(T=28.28$, constraint implementation $(\mathrm{C} 1))$.

\begin{tabular}{|c||c|c|c|}
\hline & $\sigma_{0}^{2}=0.01$ & $\sigma_{0}^{2}=0.05$ & $\sigma_{0}^{2}=0.10$ \\
\hline \hline$S E$-opt & $95.5 \%(S E=0.66 \%)$ & $92.6 \%(S E=0.83 \%)$ & $93.2 \%(S E=0.80 \%)$ \\
\hline$D$-opt & $95.7 \%(S E=0.64 \%)$ & $95.1 \%(S E=0.68 \%)$ & $93.1 \%(S E=0.80 \%)$ \\
\hline$E$-opt & $94.9 \%(S E=0.70 \%)$ & $92.8 \%(S E=0.82 \%)$ & $90.5 \%(S E=0.93 \%)$ \\
\hline Uniform & $95.3 \%(S E=0.67 \%)$ & $94.2 \%(S E=0.74 \%)$ & $93.3 \%(S E=0.79 \%)$ \\
\hline
\end{tabular}




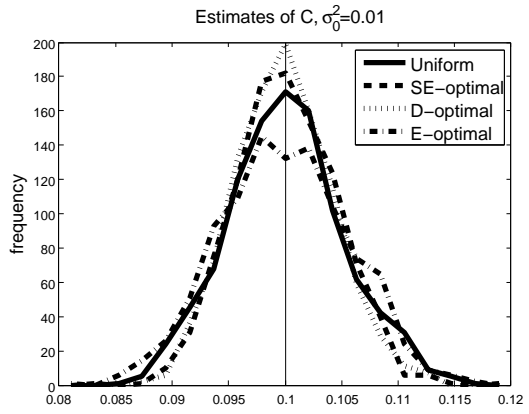

(a)

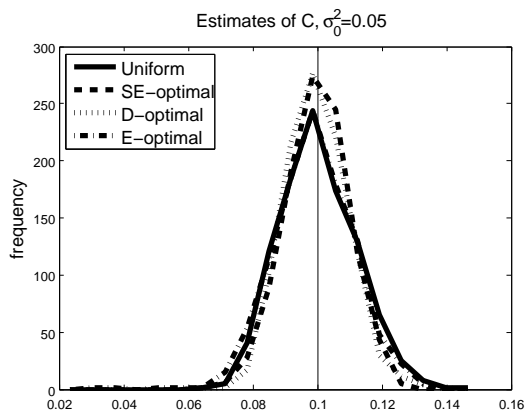

(c)

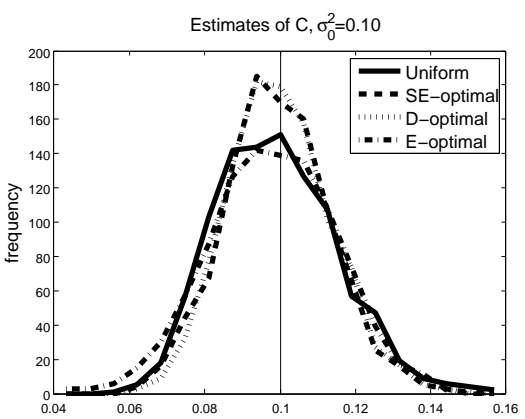

(e)

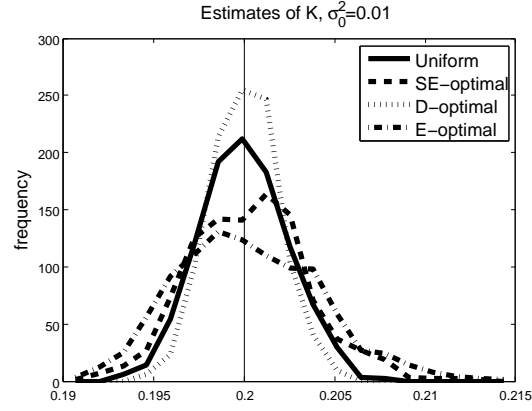

(b)

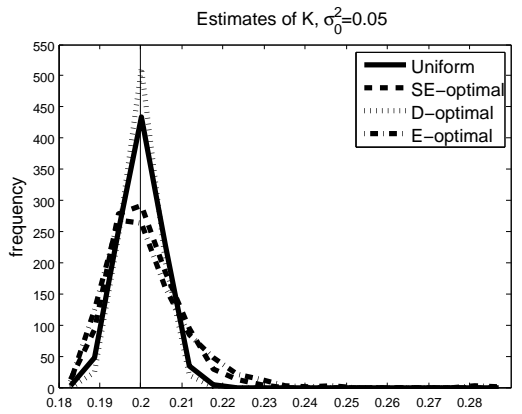

(d)

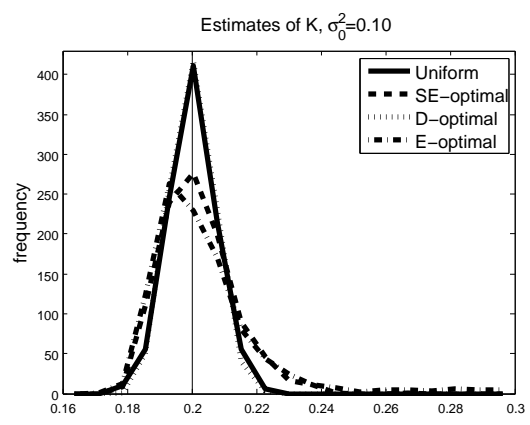

(f)

Figure 12: Histograms of parameter estimates $(\hat{C}$ in panels (a),(c),(e), and $\hat{K}$ in panels (b),(d),(f)) resulting from Monte Carlo simulation. Simulated data was generated with $\sigma_{0}^{2}=0.01$ (top row), $\sigma_{0}^{2}=0.05$ (middle row), and $\sigma_{0}^{2}=0.10$ (bottom row) ( $T=28.28$ and constraint implementation $(\mathrm{C} 1))$. 


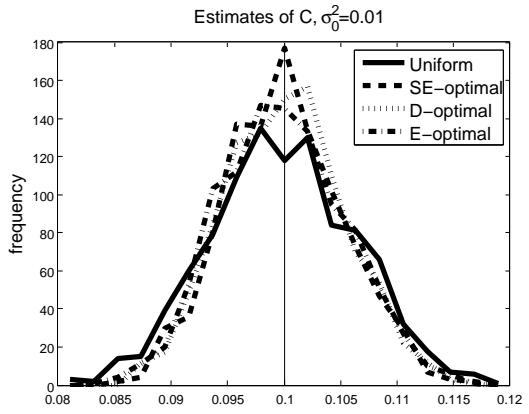

(a)

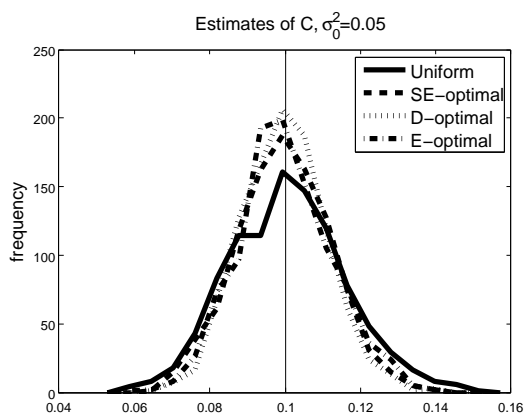

(c)

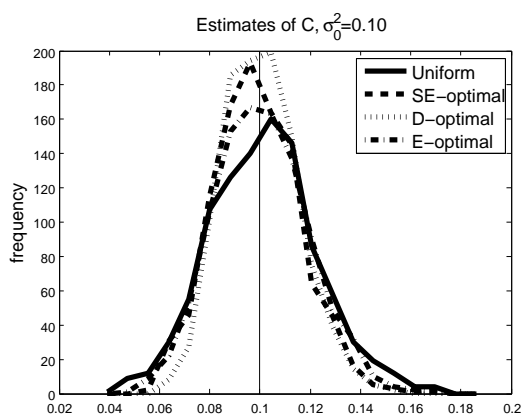

(e)

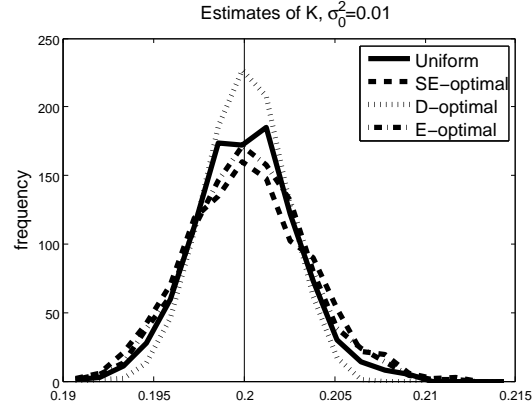

(b)

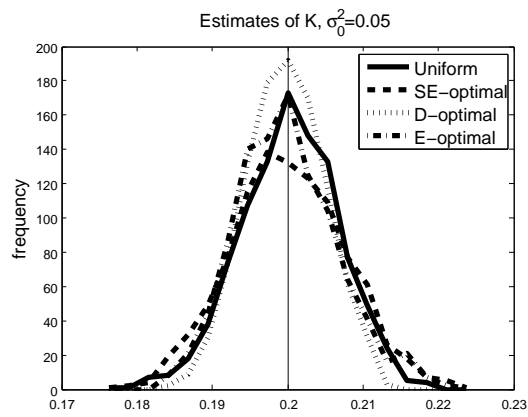

(d)

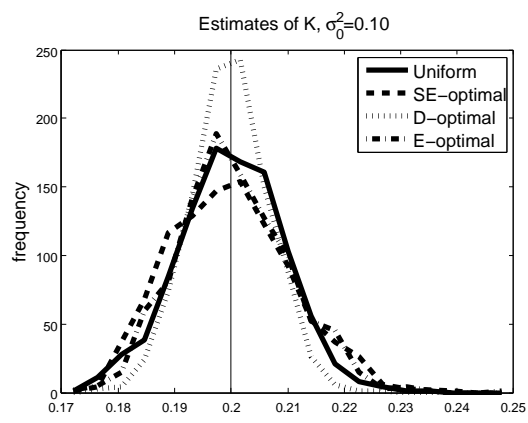

(f)

Figure 13: Histograms of parameter estimates $(\hat{C}$ in panels (a),(c),(e), and $\hat{K}$ in panels (b),(d),(f)) resulting from Monte Carlo simulation. Simulated data was generated with $\sigma_{0}^{2}=0.01$ (top row), $\sigma_{0}^{2}=0.05$ (middle row), and $\sigma_{0}^{2}=0.10$ (bottom row) ( $T=14.14$ and constraint implementation $(\mathrm{C} 2))$. 
Table 14: Average estimates $\hat{\theta}_{\text {avg }}$ with corresponding standard deviations $\hat{\theta}_{\mathrm{SD}}$ from $M=1000$ Monte Carlo trials as well as asymptotic standard errors $S E(\hat{\theta})$. Estimation based on constraint implementation (C2).

\begin{tabular}{|c|c|c|c|c|}
\hline \multicolumn{5}{|c|}{$\mathbf{T}=\mathbf{1 5}, \boldsymbol{\sigma}_{\mathbf{0}}^{\mathbf{2}}=\mathbf{0 . 0 1}$} \\
\hline \multicolumn{5}{|c|}{} \\
\hline & $S E$-opt & $D$-opt & $E$-opt & Uniform \\
\hline \hline$\hat{C}_{\text {avg }}$ & 0.1000 & 0.1000 & 0.0998 & 0.0998 \\
\hline$\hat{C}_{\text {SD }}$ & $5.264 \times 10^{-3}$ & $5.261 \times 10^{-3}$ & $5.536 \times 10^{-3}$ & $6.431 \times 10^{-3}$ \\
\hline$S E(\hat{C})$ & $5.444 \times 10^{-3}$ & $4.987 \times 10^{-3}$ & $5.700 \times 10^{-3}$ & $6.577 \times 10^{-3}$ \\
\hline$\hat{K}_{\text {avg }}$ & 0.2002 & 0.2000 & 0.2002 & 0.2000 \\
\hline$\hat{K}_{\mathrm{SD}}$ & $3.424 \times 10^{-3}$ & $2.211 \times 10^{-3}$ & $3.112 \times 10^{-3}$ & $2.867 \times 10^{-3}$ \\
\hline$S E(\hat{K})$ & $3.344 \times 10^{-3}$ & $2.181 \times 10^{-3}$ & $3.008 \times 10^{-3}$ & $2.895 \times 10^{-3}$ \\
\hline \multicolumn{5}{|c|}{$\boldsymbol{T}=\mathbf{2 8 . 2 8}$} \\
\hline$\hat{C}_{\text {avg }}$ & 0.0999 & 0.0998 & 0.0998 & 0.1001 \\
\hline$\hat{C}_{\mathrm{SD}}$ & $4.741 \times 10^{-3}$ & $4.630 \times 10^{-3}$ & $5.454 \times 10^{-3}$ & $5.342 \times 10^{-3}$ \\
\hline$S E(\hat{C})$ & $4.537 \times 10^{-3}$ & $4.528 \times 10^{-3}$ & $5.279 \times 10^{-3}$ & $5.127 \times 10^{-3}$ \\
\hline$\hat{K}_{\text {avg }}$ & 0.2001 & 0.2000 & 0.2000 & 0.2000 \\
\hline$\hat{K}_{\text {SD }}$ & $3.414 \times 10^{-3}$ & $2.202 \times 10^{-3}$ & $3.509 \times 10^{-3}$ & $2.285 \times 10^{-3}$ \\
\hline$S E(\hat{K})$ & $3.144 \times 10^{-3}$ & $2.137 \times 10^{-3}$ & $3.260 \times 10^{-3}$ & $2.307 \times 10^{-3}$ \\
\hline
\end{tabular}

Table 15: Average estimates $\hat{\theta}_{\text {avg }}$ with corresponding standard deviations $\hat{\theta}_{\mathrm{SD}}$ from $M=1000$ Monte Carlo trials as well as asymptotic standard errors $S E(\hat{\theta})$. Estimation based on constraint implementation (C2).

\begin{tabular}{|c||c|c|c|c|}
\hline \multicolumn{5}{|c|}{$\mathbf{T}=\mathbf{1 5}, \boldsymbol{\sigma}_{\mathbf{0}}^{\mathbf{2}}=\mathbf{0 . 0 5}$} \\
\hline \multicolumn{5}{|c|}{$\mathbf{1 4 . 1 4}$} \\
\hline & $S E$-opt & $D$-opt & $E$-opt & Uniform \\
\hline \hline$\hat{C}_{\text {avg }}$ & 0.0996 & 0.0997 & 0.0999 & 0.1005 \\
\hline$\hat{C}_{\mathrm{SD}}$ & $1.247 \times 10^{-2}$ & $1.125 \times 10^{-2}$ & $1.238 \times 10^{-2}$ & $1.564 \times 10^{-2}$ \\
\hline$S E(\hat{C})$ & $1.217 \times 10^{-2}$ & $1.115 \times 10^{-2}$ & $1.275 \times 10^{-2}$ & $1.471 \times 10^{-2}$ \\
\hline$\hat{K}_{\text {avg }}$ & 0.2002 & 0.1997 & 0.1999 & 0.2002 \\
\hline$\hat{K}_{\mathrm{SD}}$ & $7.715 \times 10^{-3}$ & $5.107 \times 10^{-3}$ & $6.751 \times 10^{-3}$ & $6.549 \times 10^{-3}$ \\
\hline$S E(\hat{K})$ & $7.477 \times 10^{-3}$ & $4.876 \times 10^{-3}$ & $6.727 \times 10^{-3}$ & $6.473 \times 10^{-3}$ \\
\hline \multicolumn{5}{|c|}{$\mathbf{T}=\mathbf{2 8 . 2 8}$} \\
\hline$\hat{C}_{\text {avg }}$ & 0.0990 & 0.0997 & 0.0998 & 0.1007 \\
\hline$\hat{C}_{\mathrm{SD}}$ & $1.008 \times 10^{-2}$ & $9.940 \times 10^{-3}$ & $1.172 \times 10^{-2}$ & $1.153 \times 10^{-2}$ \\
\hline$S E(\hat{C})$ & $1.015 \times 10^{-2}$ & $1.012 \times 10^{-2}$ & $1.180 \times 10^{-2}$ & $1.146 \times 10^{-2}$ \\
\hline$\hat{K}_{\text {avg }}$ & 0.2006 & 0.2000 & 0.2009 & 0.2000 \\
\hline$\hat{K}_{\mathrm{SD}}$ & $7.797 \times 10^{-3}$ & $4.753 \times 10^{-3}$ & $8.777 \times 10^{-3}$ & $5.443 \times 10^{-3}$ \\
\hline$S E(\hat{K})$ & $7.030 \times 10^{-3}$ & $4.778 \times 10^{-3}$ & $7.289 \times 10^{-3}$ & $5.159 \times 10^{-3}$ \\
\hline
\end{tabular}


Table 16: Average estimates $\hat{\theta}_{\text {avg }}$ with corresponding standard deviations $\hat{\theta}_{\mathrm{SD}}$ from $M=1000$ Monte Carlo trials as well as asymptotic standard errors $S E(\hat{\theta})$. Estimation based on constraint implementation (C2).

\begin{tabular}{|c|c|c|c|c|}
\hline \multicolumn{5}{|c|}{$\boldsymbol{T}=\mathbf{1 5}, \boldsymbol{\sigma}_{\mathbf{0}}^{\mathbf{2}}=\mathbf{0 . 1}$} \\
\hline \multicolumn{5}{|c|}{$\mathbf{1 4 . 1 4}$} \\
\hline & $S E$-opt & $D$-opt & $E$-opt & Uniform \\
\hline \hline$\hat{C}_{\text {avg }}$ & 0.0988 & 0.1002 & 0.1003 & 0.1014 \\
\hline$\hat{C}_{\text {SD }}$ & $1.703 \times 10^{-2}$ & $1.492 \times 10^{-2}$ & $1.863 \times 10^{-2}$ & $2.138 \times 10^{-2}$ \\
\hline$S E(\hat{C})$ & $1.721 \times 10^{-2}$ & $1.577 \times 10^{-2}$ & $1.802 \times 10^{-2}$ & $2.080 \times 10^{-2}$ \\
\hline$\hat{K}_{\text {avg }}$ & 0.1998 & 0.1997 & 0.2004 & 0.2000 \\
\hline$\hat{K}_{\mathrm{SD}}$ & $1.073 \times 10^{-2}$ & $6.885 \times 10^{-3}$ & $9.921 \times 10^{-3}$ & $9.483 \times 10^{-3}$ \\
\hline$S E(\hat{K})$ & $1.057 \times 10^{-2}$ & $6.895 \times 10^{-3}$ & $9.513 \times 10^{-3}$ & $9.154 \times 10^{-3}$ \\
\hline \multicolumn{5}{|c|}{$\mathbf{T}=\mathbf{2 8 . 2 8}$} \\
\hline$\hat{C}_{\text {avg }}$ & 0.0990 & 0.0997 & 0.0998 & 0.1007 \\
\hline$\hat{C}_{\mathrm{SD}}$ & $1.008 \times 10^{-2}$ & $9.940 \times 10^{-3}$ & $1.172 \times 10^{-2}$ & $1.153 \times 10^{-2}$ \\
\hline$S E(\hat{C})$ & $1.015 \times 10^{-2}$ & $1.012 \times 10^{-2}$ & $1.180 \times 10^{-2}$ & $1.146 \times 10^{-2}$ \\
\hline$\hat{K}_{\text {avg }}$ & 0.2006 & 0.2000 & 0.2009 & 0.2000 \\
\hline$\hat{K}_{\text {SD }}$ & $7.797 \times 10^{-3}$ & $4.753 \times 10^{-3}$ & $8.777 \times 10^{-3}$ & $5.443 \times 10^{-3}$ \\
\hline$S E(\hat{K})$ & $7.030 \times 10^{-3}$ & $4.778 \times 10^{-3}$ & $7.289 \times 10^{-3}$ & $5.159 \times 10^{-3}$ \\
\hline
\end{tabular}

Table 17: Percent of confidence ellipsoids which contain the true parameter values $(T=14.14$, constraint implementation $(\mathrm{C} 2))$.

\begin{tabular}{|c||c|c|c|}
\hline & $\sigma_{0}^{2}=0.01$ & $\sigma_{0}^{2}=0.05$ & $\sigma_{0}^{2}=0.10$ \\
\hline \hline$S E$-opt & $95.5 \%(S E=0.66 \%)$ & $93.9 \%(S E=0.76 \%)$ & $94.3 \%(S E=0.73 \%)$ \\
\hline$D$-opt & $95.3 \%(S E=0.67 \%)$ & $93.7 \%(S E=0.77 \%)$ & $95.5 \%(S E=0.66 \%)$ \\
\hline$E$-opt & $94.6 \%(S E=0.71 \%)$ & $94.9 \%(S E=0.70 \%)$ & $94.0 \%(S E=0.75 \%)$ \\
\hline Uniform & $95.1 \%(S E=0.68 \%)$ & $95.1 \%(S E=0.68 \%)$ & $94.5 \%(S E=0.72 \%)$ \\
\hline
\end{tabular}

Table 18: Percent of confidence ellipsoids which contain the true parameter values $(T=28.28$, constraint implementation $(\mathrm{C} 2))$.

\begin{tabular}{|c||c|c|c|}
\hline & $\sigma_{0}^{2}=0.01$ & $\sigma_{0}^{2}=0.05$ & $\sigma_{0}^{2}=0.10$ \\
\hline \hline$S E$-opt & $94.6 \%(S E=0.71 \%)$ & $93.4 \%(S E=0.79 \%)$ & $93.1 \%(S E=0.80 \%)$ \\
\hline$D$-opt & $93.7 \%(S E=0.77 \%)$ & $95.0 \%(S E=0.69 \%)$ & $92.3 \%(S E=0.84 \%)$ \\
\hline$E$-opt & $93.0 \%(S E=0.81 \%)$ & $92.7 \%(S E=0.82 \%)$ & $92.4 \%(S E=0.84 \%)$ \\
\hline Uniform & $94.6 \%(S E=0.71 \%)$ & $94.9 \%(S E=0.70 \%)$ & $95.2 \%(S E=0.68 \%)$ \\
\hline
\end{tabular}




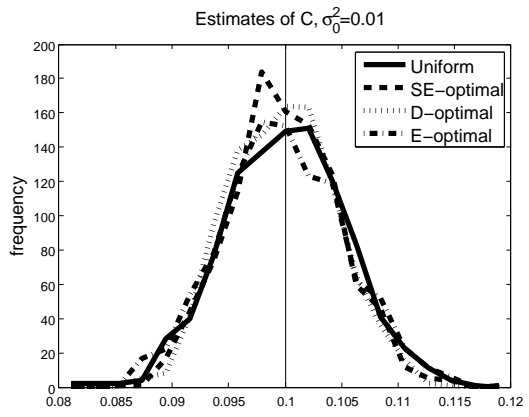

(a)

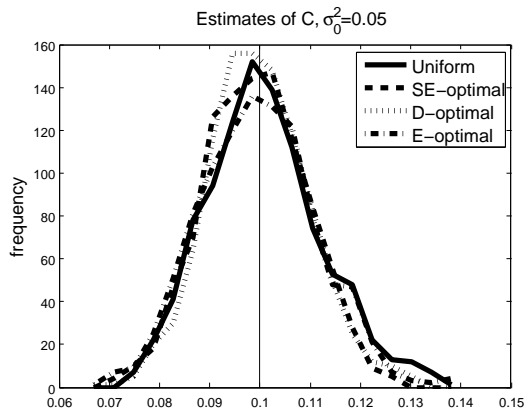

(c)

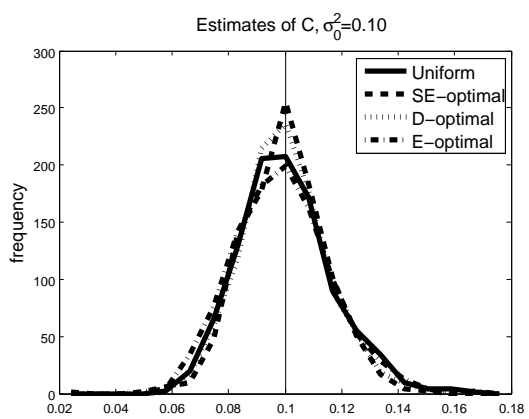

(e)

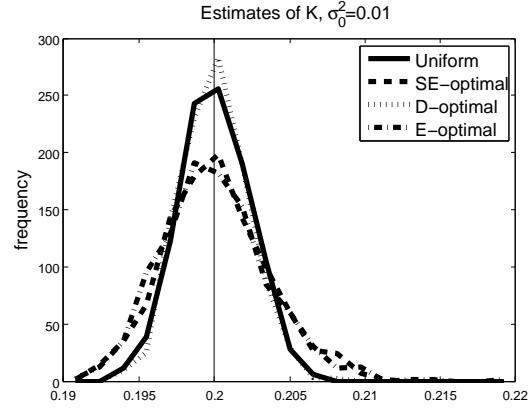

(b)

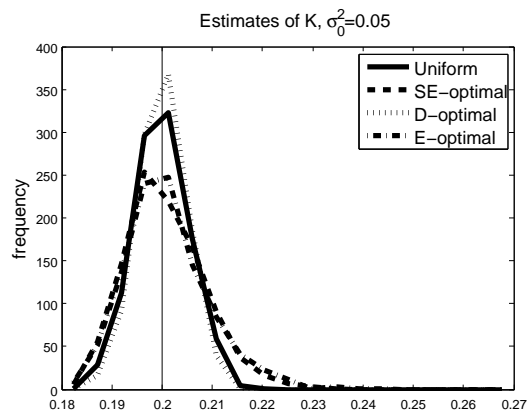

(d)

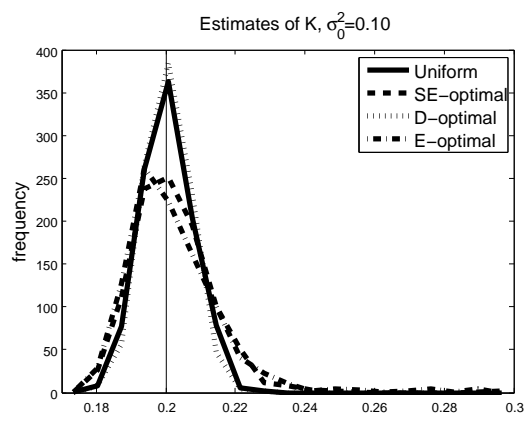

(f)

Figure 14: Histograms of parameter estimates $(\hat{C}$ in panels (a),(c),(e), and $\hat{K}$ in panels (b),(d),(f)) resulting from Monte Carlo simulation. Simulated data was generated with $\sigma_{0}^{2}=0.01$ (top row), $\sigma_{0}^{2}=0.05$ (middle row), and $\sigma_{0}^{2}=0.10$ (bottom row) ( $T=28.28$ and constraint implementation $(\mathrm{C} 2))$. 


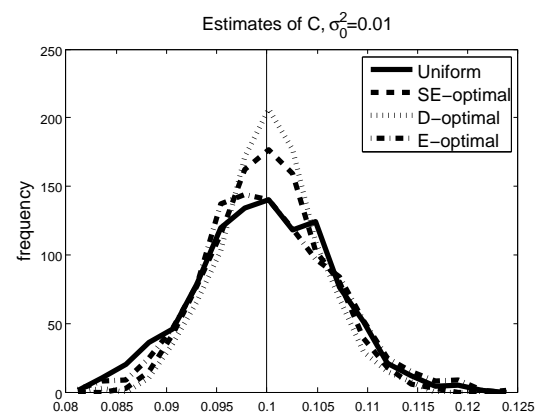

(a)

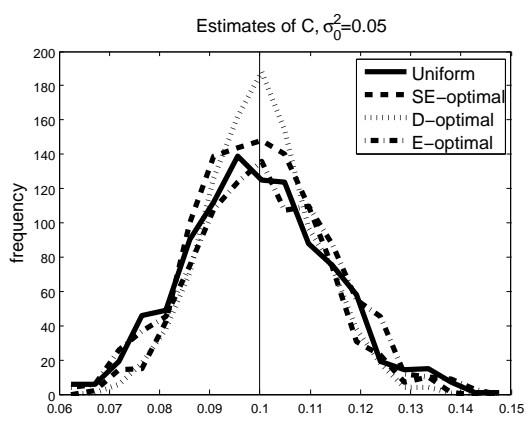

(c)

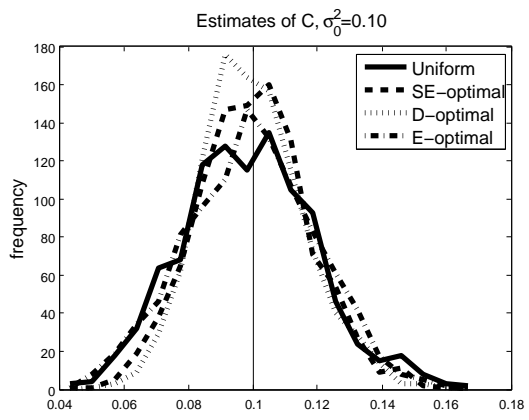

(e)

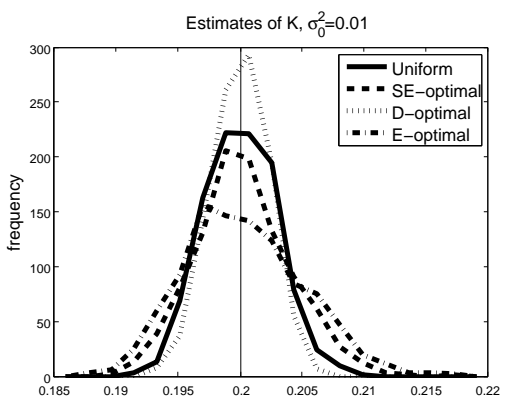

(b)

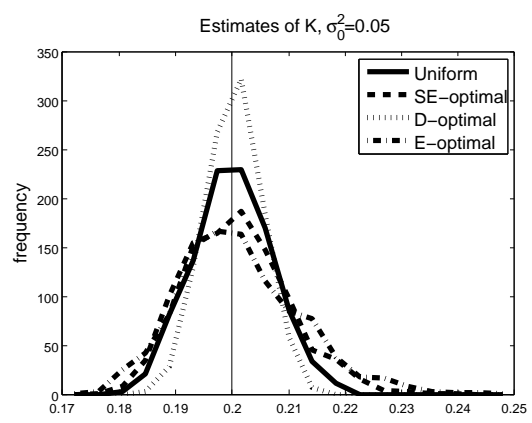

(d)

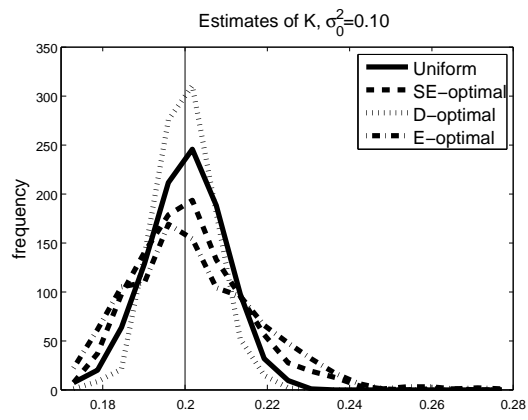

(f)

Figure 15: Histograms of parameter estimates $(\hat{C}$ in panels (a),(c),(e), and $\hat{K}$ in panels (b),(d),(f)) resulting from Monte Carlo simulation. Simulated data was generated with $\sigma_{0}^{2}=0.01$ (top row), $\sigma_{0}^{2}=0.05$ (middle row), and $\sigma_{0}^{2}=0.10$ (bottom row) $(T=14.14$ and constraint implementation (C3)). 
Table 19: Average estimates $\hat{\theta}_{\text {avg }}$ with corresponding standard deviations $\hat{\theta}_{\mathrm{SD}}$ from $M=1000$ Monte Carlo trials as well as asymptotic standard errors $S E(\hat{\theta})$. Estimation based on constraint implementation (C3).

\begin{tabular}{|c||c|c|c|c|}
\hline \multicolumn{5}{|c|}{$\boldsymbol{T}=\mathbf{1 5}, \boldsymbol{\sigma}_{\mathbf{0}}^{\mathbf{2}}=\mathbf{0 . 0 1}$} \\
\hline \multicolumn{5}{|c|}{$\mathbf{1 4 . 1 4}$} \\
\hline & $S E$-opt & $D$-opt & $E$-opt & Uniform \\
\hline \hline$\hat{C}_{\text {avg }}$ & 0.1002 & 0.1002 & 0.1003 & 0.0999 \\
\hline$\hat{C}_{\mathrm{SD}}$ & $5.396 \times 10^{-3}$ & $4.957 \times 10^{-3}$ & $6.616 \times 10^{-3}$ & $6.664 \times 10^{-3}$ \\
\hline$S E(\hat{C})$ & $5.124 \times 10^{-3}$ & $4.938 \times 10^{-3}$ & $6.361 \times 10^{-3}$ & $6.577 \times 10^{-3}$ \\
\hline$\hat{K}_{\text {avg }}$ & 0.2002 & 0.2000 & 0.2002 & 0.2000 \\
\hline$\hat{K}_{\mathrm{SD}}$ & $3.888 \times 10^{-3}$ & $2.318 \times 10^{-3}$ & $4.770 \times 10^{-3}$ & $2.888 \times 10^{-3}$ \\
\hline$S E(\hat{K})$ & $3.735 \times 10^{-3}$ & $2.248 \times 10^{-3}$ & $4.162 \times 10^{-3}$ & $2.895 \times 10^{-3}$ \\
\hline \multicolumn{5}{|c|}{$\mathbf{T}=\mathbf{2 8 . 2 8}$} \\
\hline$\hat{C}_{\text {avg }}$ & 0.0999 & 0.0999 & 0.1002 & 0.1000 \\
\hline$\hat{C}_{\mathrm{SD}}$ & $4.785 \times 10^{-3}$ & $4.398 \times 10^{-3}$ & $5.593 \times 10^{-3}$ & $5.147 \times 10^{-3}$ \\
\hline$S E(\hat{C})$ & $4.492 \times 10^{-3}$ & $4.372 \times 10^{-3}$ & $5.409 \times 10^{-3}$ & $5.127 \times 10^{-3}$ \\
\hline$\hat{K}_{\text {avg }}$ & 0.2001 & 0.1999 & 0.2005 & 0.1999 \\
\hline$\hat{K}_{\mathrm{SD}}$ & $3.325 \times 10^{-3}$ & $2.027 \times 10^{-3}$ & $4.067 \times 10^{-3}$ & $2.317 \times 10^{-3}$ \\
\hline$S E(\hat{K})$ & $3.088 \times 10^{-3}$ & $2.005 \times 10^{-3}$ & $3.539 \times 10^{-3}$ & $2.307 \times 10^{-3}$ \\
\hline
\end{tabular}

Table 20: Average estimates $\hat{\theta}_{\text {avg }}$ with corresponding standard deviations $\hat{\theta}_{\mathrm{SD}}$ from $M=1000$ Monte Carlo trials as well as asymptotic standard errors $S E(\hat{\theta})$. Estimation based on constraint implementation (C3).

\begin{tabular}{|c||c|c|c|c|}
\hline \multicolumn{5}{|c|}{$\mathbf{T}=\mathbf{1 5}, \boldsymbol{\sigma}_{\mathbf{0}}^{\mathbf{2}}=\mathbf{0 . 0 5}$} \\
\hline \multicolumn{5}{|c|}{$\mathbf{1 4 . 1 4}$} \\
\hline & $S E$-opt & $D$-opt & $E$-opt & Uniform \\
\hline \hline$\hat{C}_{\text {avg }}$ & 0.0997 & 0.1001 & 0.1008 & 0.0996 \\
\hline$\hat{C}_{\text {SD }}$ & $1.185 \times 10^{-2}$ & $1.082 \times 10^{-2}$ & $1.451 \times 10^{-2}$ & $1.474 \times 10^{-2}$ \\
\hline$S E(\hat{C})$ & $1.146 \times 10^{-2}$ & $1.104 \times 10^{-2}$ & $1.422 \times 10^{-2}$ & $1.471 \times 10^{-2}$ \\
\hline$\hat{K}_{\text {avg }}$ & 0.2007 & 0.2002 & 0.2014 & 0.2000 \\
\hline$\hat{K}_{\text {SD }}$ & $9.063 \times 10^{-3}$ & $4.951 \times 10^{-3}$ & $1.089 \times 10^{-2}$ & $6.885 \times 10^{-3}$ \\
\hline$S E(\hat{K})$ & $8.351 \times 10^{-3}$ & $5.028 \times 10^{-3}$ & $9.307 \times 10^{-3}$ & $6.473 \times 10^{-3}$ \\
\hline \multicolumn{5}{|c|}{$\mathbf{T}=\mathbf{2 8 . 2 8}$} \\
\hline$\hat{C}_{\text {avg }}$ & 0.0993 & 0.1000 & 0.0991 & 0.0992 \\
\hline$\hat{C}_{\text {SD }}$ & $1.010 \times 10^{-2}$ & $9.973 \times 10^{-3}$ & $1.313 \times 10^{-2}$ & $1.147 \times 10^{-2}$ \\
\hline$S E(\hat{C})$ & $1.005 \times 10^{-2}$ & $9.775 \times 10^{-3}$ & $1.209 \times 10^{-2}$ & $1.146 \times 10^{-2}$ \\
\hline$\hat{K}_{\text {avg }}$ & 0.2006 & 0.2001 & 0.2027 & 0.2003 \\
\hline$\hat{K}_{\text {SD }}$ & $7.581 \times 10^{-3}$ & $4.465 \times 10^{-3}$ & $1.433 \times 10^{-2}$ & $5.200 \times 10^{-3}$ \\
\hline$S E(\hat{K})$ & $6.905 \times 10^{-3}$ & $4.484 \times 10^{-3}$ & $7.913 \times 10^{-3}$ & $5.159 \times 10^{-3}$ \\
\hline
\end{tabular}


Table 21: Average estimates $\hat{\theta}_{\text {avg }}$ with corresponding standard deviations $\hat{\theta}_{\mathrm{SD}}$ from $M=1000$ Monte Carlo trials as well as asymptotic standard errors $S E(\hat{\theta})$. Estimation based on constraint implementation (C3).

\begin{tabular}{|c||c|c|c|c|}
\hline \multicolumn{5}{|c|}{$\mathbf{T}=\mathbf{1 5}, \boldsymbol{\sigma}_{\mathbf{0}}^{\mathbf{2}}=\mathbf{0 . 1}$} \\
\hline \multicolumn{5}{|c|}{} \\
\hline & $S E$-opt & $D$-opt & $E$-opt & Uniform \\
\hline \hline$\hat{C}_{\text {avg }}$ & 0.1002 & 0.1001 & 0.0995 & 0.0988 \\
\hline$\hat{C}_{\text {SD }}$ & $1.736 \times 10^{-2}$ & $1.603 \times 10^{-2}$ & $2.028 \times 10^{-2}$ & $2.074 \times 10^{-2}$ \\
\hline$S E(\hat{C})$ & $1.620 \times 10^{-2}$ & $1.562 \times 10^{-2}$ & $2.012 \times 10^{-2}$ & $2.080 \times 10^{-2}$ \\
\hline$\hat{K}_{\text {avg }}$ & 0.2012 & 0.2001 & 0.2021 & 0.2004 \\
\hline$\hat{K}_{\mathrm{SD}}$ & $1.310 \times 10^{-2}$ & $7.205 \times 10^{-3}$ & $1.665 \times 10^{-2}$ & $9.417 \times 10^{-3}$ \\
\hline$S E(\hat{K})$ & $1.181 \times 10^{-2}$ & $7.110 \times 10^{-3}$ & $1.316 \times 10^{-2}$ & $9.154 \times 10^{-3}$ \\
\hline \multicolumn{5}{|c|}{$\boldsymbol{T}=\mathbf{2 8 . 2 8}$} \\
\hline$\hat{C}_{\text {avg }}$ & 0.0989 & 0.1001 & 0.0968 & 0.1007 \\
\hline$\hat{C}_{\mathrm{SD}}$ & $1.448 \times 10^{-2}$ & $1.372 \times 10^{-2}$ & $1.743 \times 10^{-2}$ & $1.730 \times 10^{-2}$ \\
\hline$S E(\hat{C})$ & $1.421 \times 10^{-2}$ & $1.382 \times 10^{-2}$ & $1.710 \times 10^{-2}$ & $1.621 \times 10^{-2}$ \\
\hline$\hat{K}_{\text {avg }}$ & 0.2019 & 0.2002 & 0.2056 & 0.2002 \\
\hline$\hat{K}_{\mathrm{SD}}$ & $1.218 \times 10^{-2}$ & $6.432 \times 10^{-3}$ & $2.156 \times 10^{-2}$ & $7.437 \times 10^{-3}$ \\
\hline$S E(\hat{K})$ & $9.765 \times 10^{-3}$ & $6.341 \times 10^{-3}$ & $1.119 \times 10^{-2}$ & $7.295 \times 10^{-3}$ \\
\hline
\end{tabular}


Table 22: Percent of confidence ellipsoids which contain the true parameter values $(T=14.14$, constraint implementation $(\mathrm{C} 3))$.

\begin{tabular}{|c||c|c|c|}
\hline & $\sigma_{0}^{2}=0.01$ & $\sigma_{0}^{2}=0.05$ & $\sigma_{0}^{2}=0.10$ \\
\hline \hline$S E$-opt & $94.7 \%(S E=0.71 \%)$ & $94.9 \%(S E=0.70 \%)$ & $93.5 \%(S E=0.78 \%)$ \\
\hline$D$-opt & $93.9 \%(S E=0.76 \%)$ & $95.1 \%(S E=0.68 \%)$ & $95.1 \%(S E=0.68 \%)$ \\
\hline$E$-opt & $94.9 \%(S E=0.70 \%)$ & $93.8 \%(S E=0.76 \%)$ & $92.1 \%(S E=0.85 \%)$ \\
\hline Uniform & $94.3 \%(S E=0.73 \%)$ & $93.7 \%(S E=0.77 \%)$ & $95.6 \%(S E=0.65 \%)$ \\
\hline
\end{tabular}

Table 23: Percent of confidence ellipsoids which contain the true parameter values $(T=28.28$, constraint implementation $(\mathrm{C} 3))$.

\begin{tabular}{|c||c|c|c|}
\hline & $\sigma_{0}^{2}=0.01$ & $\sigma_{0}^{2}=0.05$ & $\sigma_{0}^{2}=0.10$ \\
\hline \hline$S E$-opt & $93.9 \%(S E=0.76 \%)$ & $94.6 \%(S E=0.71 \%)$ & $93.3 \%(S E=0.79 \%)$ \\
\hline$D$-opt & $94.4 \%(S E=0.73 \%)$ & $94.7 \%(S E=0.71 \%)$ & $94.6 \%(S E=0.71 \%)$ \\
\hline$E$-opt & $95.3 \%(S E=0.67 \%)$ & $91.1 \%(S E=0.90 \%)$ & $86.5 \%(S E=1.08 \%)$ \\
\hline Uniform & $94.7 \%(S E=0.71 \%)$ & $95.3 \%(S E=0.67 \%)$ & $93.9 \%(S E=0.76 \%)$ \\
\hline
\end{tabular}

Table 24: Average estimates $\hat{\theta}_{\text {avg }}$ with corresponding standard deviations $\hat{\theta}_{\mathrm{SD}}$ from $M=1000$ Monte Carlo trials as well as asymptotic standard errors $S E(\hat{\theta})$. Estimation based on constraint implementation (C4).

\begin{tabular}{|c||c|c|c|c|}
\hline \multicolumn{5}{|c|}{$\boldsymbol{T}=\mathbf{1 5}, \boldsymbol{\sigma}_{\mathbf{0}}^{\mathbf{2}}=\mathbf{0 . 0 1}$} \\
\hline \multicolumn{5}{|c|}{$\mathbf{1 4 . 1 4}^{-1}$} \\
\hline & $S E$-opt & $D$-opt & $E$-opt & Uniform \\
\hline \hline$\hat{C}_{\text {avg }}$ & 0.0999 & 0.1003 & 0.0998 & 0.0998 \\
\hline$\hat{C}_{\mathrm{SD}}$ & $5.235 \times 10^{-3}$ & $5.136 \times 10^{-3}$ & $6.716 \times 10^{-3}$ & $6.532 \times 10^{-3}$ \\
\hline$S E(\hat{C})$ & $5.124 \times 10^{-3}$ & $5.108 \times 10^{-3}$ & $6.361 \times 10^{-3}$ & $6.577 \times 10^{-3}$ \\
\hline$\hat{K}_{\text {avg }}$ & 0.2001 & 0.2000 & 0.2000 & 0.2001 \\
\hline$\hat{K}_{\mathrm{SD}}$ & $4.010 \times 10^{-3}$ & $2.289 \times 10^{-3}$ & $4.426 \times 10^{-3}$ & $2.926 \times 10^{-3}$ \\
\hline$S E(\hat{K})$ & $3.735 \times 10^{-3}$ & $2.295 \times 10^{-3}$ & $4.162 \times 10^{-3}$ & $2.895 \times 10^{-3}$ \\
\hline \multicolumn{5}{|c|}{$\boldsymbol{T}=\mathbf{2 8 . 2 8}$} \\
\hline$\hat{C}_{\text {avg }}$ & 0.0999 & 0.1001 & 0.1001 & 0.1000 \\
\hline$\hat{C}_{\mathrm{SD}}$ & $4.434 \times 10^{-3}$ & $4.303 \times 10^{-3}$ & $5.596 \times 10^{-3}$ & $4.906 \times 10^{-3}$ \\
\hline$S E(\hat{C})$ & $4.504 \times 10^{-3}$ & $4.391 \times 10^{-3}$ & $5.419 \times 10^{-3}$ & $5.127 \times 10^{-3}$ \\
\hline$\hat{K}_{\text {avg }}$ & 0.2001 & 0.1998 & 0.2002 & 0.1999 \\
\hline$\hat{K}_{\mathrm{SD}}$ & $3.354 \times 10^{-3}$ & $1.989 \times 10^{-3}$ & $3.924 \times 10^{-3}$ & $2.371 \times 10^{-3}$ \\
\hline$S E(\hat{K})$ & $3.116 \times 10^{-3}$ & $2.003 \times 10^{-3}$ & $3.546 \times 10^{-3}$ & $2.307 \times 10^{-3}$ \\
\hline
\end{tabular}




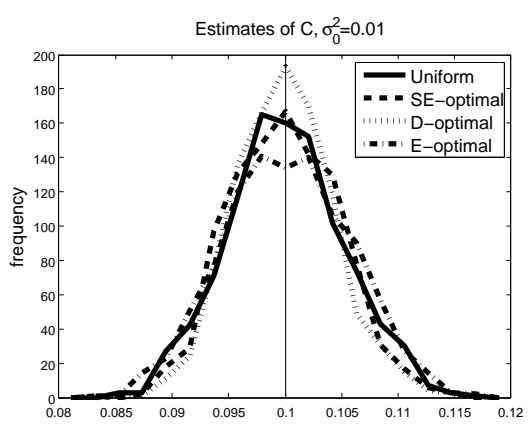

(a)

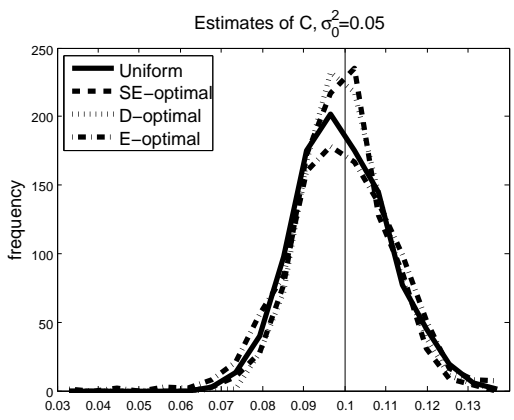

(c)

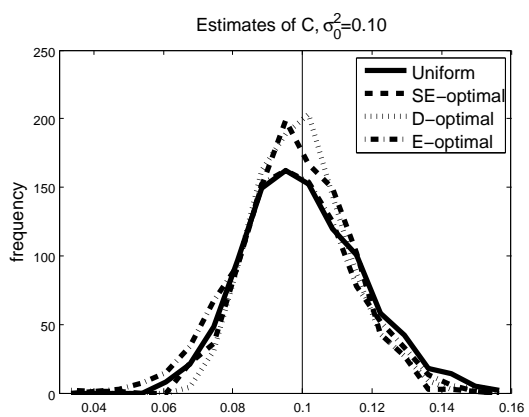

(e)

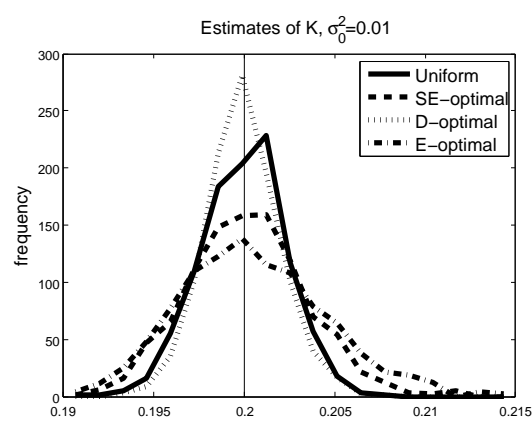

(b)

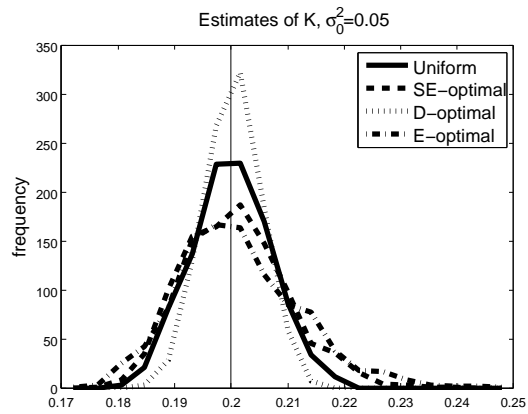

(d)

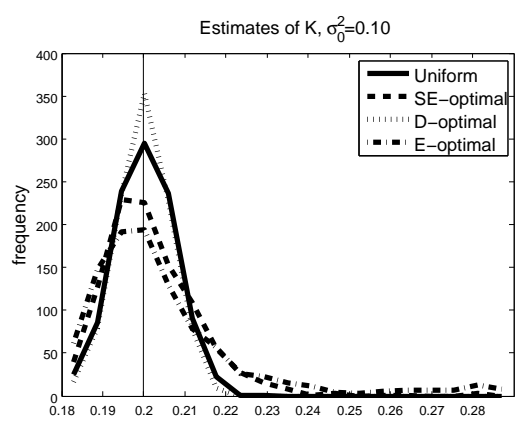

(f)

Figure 16: Histograms of parameter estimates $(\hat{C}$ in panels (a),(c),(e), and $\hat{K}$ in panel (b),(d),(f)) resulting from Monte Carlo simulation. Simulated data was generated with $\sigma_{0}^{2}=0.01$ (top row), $\sigma_{0}^{2}=0.05$ (middle row), and $\sigma_{0}^{2}=0.10$ (bottom row) $(T=28.28$ and constraint implementation (C3)).

\subsection{Discussion of Harmonic Oscillator Monte Carlo Results}

From the harmonic oscillator Monte Carlo results we again observe that as the level of noise in the data decreases so does the variance in the parameter estimates $\left(\hat{\theta}_{\mathrm{SD}}\right)$ and the estimates approach the true values. Examining the average parameter estimates, we find that in all cases the averages are close to the true values $\theta_{0}=\left(C_{\text {nom }}, K_{\text {nom }}\right)^{\top}=(0.1,0.2)^{\top}$. However in Figures $11-18$ often it 


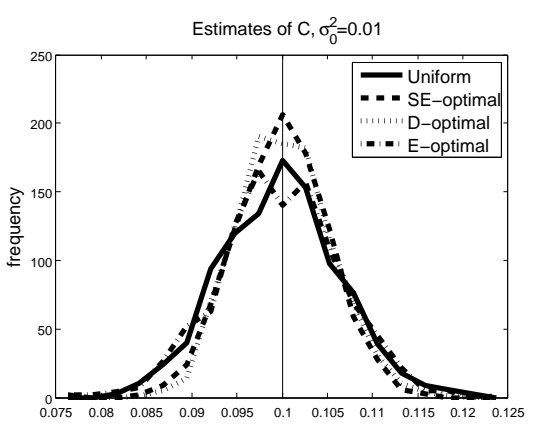

(a)

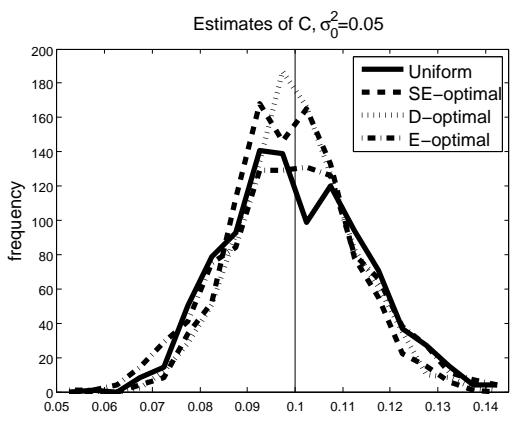

(c)

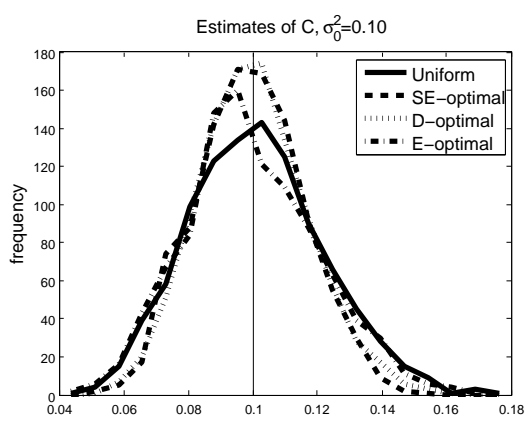

(e)

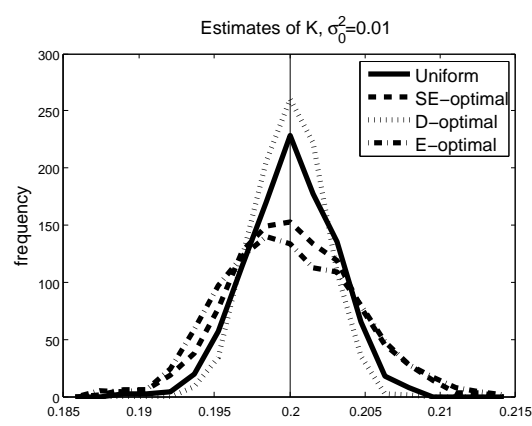

(b)

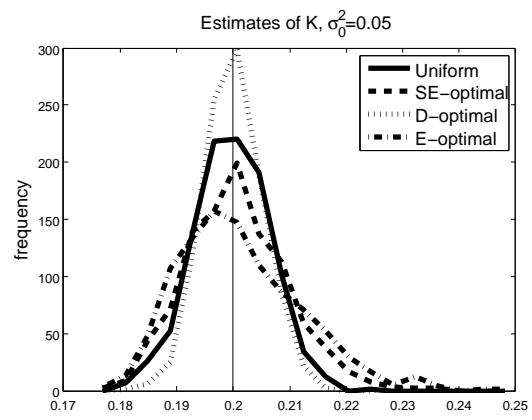

(d)

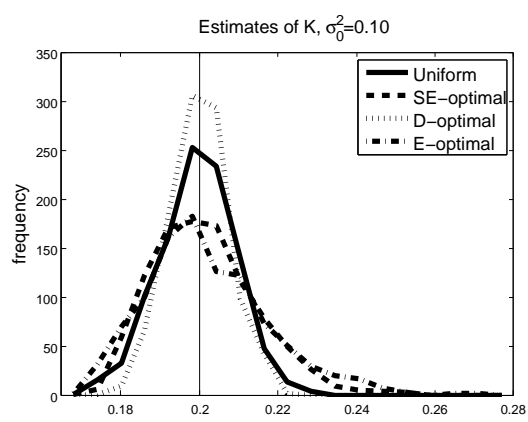

(f)

Figure 17: Histograms of parameter estimates ( $\hat{C}$ in panels (a),(c),(e), and $\hat{K}$ in panels (b),(d),(f)) resulting from Monte Carlo simulation. Simulated data was generated with $\sigma_{0}^{2}=0.01$ (top row), $\sigma_{0}^{2}=0.05$ (middle row), and $\sigma_{0}^{2}=0.10$ (bottom row) $(T=14.14$ and constraint implementation $(\mathrm{C} 4))$.

appears as though for noise levels $\sigma_{0}^{2}=0.05$ and $0.10, E$-optimal and $S E$-optimal distributions are right-skewed for the parameter $K$. Also, often $E$-optimal distributions appear to be left-skewed for parameter $C$ for the higher levels of noise in the data. This causes the average estimates of $K$ to be slightly larger than the true value for $E$-optimal and $S E$-optimal, and the average estimate of $C$ to be slightly smaller than the true value for $E$-optimal.

Comparing the standard deviations of the parameter estimates $\hat{\theta}_{\mathrm{SD}}$ to the asymptotic standard 
Table 25: Average estimates $\hat{\theta}_{\text {avg }}$ with corresponding standard deviations $\hat{\theta}_{\mathrm{SD}}$ from $M=1000$ Monte Carlo trials as well as asymptotic standard errors $S E(\hat{\theta})$. Estimation based on constraint implementation (C4).

\begin{tabular}{|c||c|c|c|c|}
\hline \multicolumn{5}{|c|}{$\boldsymbol{T}=\mathbf{1 5}, \boldsymbol{\sigma}_{\mathbf{0}}^{\mathbf{2}}=\mathbf{0 . 0 5}$} \\
\hline \multicolumn{5}{|c|}{$\mathbf{1 4 . 1 4}$} \\
\hline & $S E$-opt & $D$-opt & $E$-opt & Uniform \\
\hline \hline$\hat{C}_{\text {avg }}$ & 0.0994 & 0.1004 & 0.1000 & 0.1004 \\
\hline$\hat{C}_{\text {SD }}$ & $1.172 \times 10^{-2}$ & $1.196 \times 10^{-2}$ & $1.498 \times 10^{-2}$ & $1.459 \times 10^{-2}$ \\
\hline$S E(\hat{C})$ & $1.146 \times 10^{-2}$ & $1.142 \times 10^{-2}$ & $1.422 \times 10^{-2}$ & $1.471 \times 10^{-2}$ \\
\hline$\hat{K}_{\text {avg }}$ & 0.2006 & 0.2000 & 0.2011 & 0.1996 \\
\hline$\hat{K}_{\text {SD }}$ & $9.001 \times 10^{-3}$ & $5.172 \times 10^{-3}$ & $1.100 \times 10^{-2}$ & $6.683 \times 10^{-3}$ \\
\hline$S E(\hat{K})$ & $8.351 \times 10^{-3}$ & $5.133 \times 10^{-3}$ & $9.307 \times 10^{-3}$ & $6.473 \times 10^{-3}$ \\
\hline \multicolumn{5}{|c|}{$\mathbf{T}=\mathbf{2 8 . 2 8}$} \\
\hline$\hat{C}_{\text {avg }}$ & 0.0997 & 0.0997 & 0.0991 & 0.1000 \\
\hline$\hat{C}_{\mathrm{SD}}$ & $9.840 \times 10^{-3}$ & $1.019 \times 10^{-2}$ & $1.299 \times 10^{-2}$ & $1.147 \times 10^{-2}$ \\
\hline$S E(\hat{C})$ & $1.007 \times 10^{-2}$ & $9.820 \times 10^{-3}$ & $1.212 \times 10^{-2}$ & $1.146 \times 10^{-2}$ \\
\hline$\hat{K}_{\text {avg }}$ & 0.2007 & 0.2000 & 0.2022 & 0.1999 \\
\hline$\hat{K}_{\mathrm{SD}}$ & $7.336 \times 10^{-3}$ & $4.351 \times 10^{-3}$ & $1.268 \times 10^{-2}$ & $5.005 \times 10^{-3}$ \\
\hline$S E(\hat{K})$ & $6.967 \times 10^{-3}$ & $4.478 \times 10^{-3}$ & $7.928 \times 10^{-3}$ & $5.159 \times 10^{-3}$ \\
\hline
\end{tabular}

errors $S E(\hat{\theta})$ we find that they are very close in every case. Comparing the standard deviations of the parameter estimates among the optimal design methods and the uniform mesh often we find that $D$-optimal had the smallest standard deviations, followed by $S E$-optimal for parameter $C$ and the uniform mesh for parameter $K$. In Tables $14-16$ for $T=28.28$, and $24-26 D$-optimal and $S E$-optimal had the smallest standard deviations for the parameter $C$. For parameter $K, D$-optimal had the smallest standard deviation again followed by the uniform mesh.

For the harmonic oscillator example 95\% confidence ellipsoid coverage of the true parameter value is better overall than in the logistic example. This may not be a reasonable comparison since only $p=2$ parameters are estimated for the harmonic oscillator example, whereas in the logistic example $p=3$. In this example, no optimal design mesh is consistently better in terms of confidence ellipsoid coverage of the true parameter value. However, in almost every case $E$-optimal has the worst coverage compared to the other optimal design methods (though still being fairy decent). 
Table 26: Average estimates $\hat{\theta}_{\text {avg }}$ with corresponding standard deviations $\hat{\theta}_{\mathrm{SD}}$ from $M=1000$ Monte Carlo trials as well as asymptotic standard errors $S E(\hat{\theta})$. Estimation based on constraint implementation (C4).

\begin{tabular}{|c||c|c|c|c|}
\hline \multicolumn{5}{|c|}{$\boldsymbol{N}=\mathbf{1 5}, \boldsymbol{\sigma}_{\mathbf{0}}^{\mathbf{2}}=\mathbf{0 . 1}$} \\
\hline \multicolumn{5}{|c|}{$\mathbf{1 4 . 1 4}$} \\
\hline & $S E$-opt & $D$-opt & $E$-opt & Uniform \\
\hline \hline$\hat{C}_{\text {avg }}$ & 0.0992 & 0.1002 & 0.0996 & 0.1013 \\
\hline$\hat{C}_{\mathrm{SD}}$ & $1.635 \times 10^{-2}$ & $1.691 \times 10^{-2}$ & $2.099 \times 10^{-2}$ & $2.089 \times 10^{-2}$ \\
\hline$S E(\hat{C})$ & $1.621 \times 10^{-2}$ & $1.615 \times 10^{-2}$ & $2.012 \times 10^{-2}$ & $2.080 \times 10^{-2}$ \\
\hline$\hat{K}_{\text {avg }}$ & 0.2013 & 0.2000 & 0.2020 & 0.1994 \\
\hline$\hat{K}_{\mathrm{SD}}$ & $1.326 \times 10^{-2}$ & $7.446 \times 10^{-3}$ & $1.611 \times 10^{-2}$ & $9.633 \times 10^{-3}$ \\
\hline$S E(\hat{K})$ & $1.181 \times 10^{-2}$ & $7.259 \times 10^{-3}$ & $1.316 \times 10^{-2}$ & $9.154 \times 10^{-3}$ \\
\hline \multicolumn{5}{|c|}{$\mathbf{T}=\mathbf{2 8 . 2 8}$} \\
\hline$\hat{C}_{\text {avg }}$ & 0.0991 & 0.1004 & 0.0979 & 0.0995 \\
\hline$\hat{C}_{\mathrm{SD}}$ & $1.404 \times 10^{-2}$ & $1.377 \times 10^{-2}$ & $1.752 \times 10^{-2}$ & $1.579 \times 10^{-2}$ \\
\hline$S E(\hat{C})$ & $1.424 \times 10^{-2}$ & $1.389 \times 10^{-2}$ & $1.714 \times 10^{-2}$ & $1.621 \times 10^{-2}$ \\
\hline$\hat{K}_{\text {avg }}$ & 0.2012 & 0.2002 & 0.2046 & 0.2000 \\
\hline$\hat{K}_{\mathrm{SD}}$ & $1.168 \times 10^{-2}$ & $6.757 \times 10^{-3}$ & $1.960 \times 10^{-2}$ & $7.242 \times 10^{-3}$ \\
\hline$S E(\hat{K})$ & $9.853 \times 10^{-3}$ & $6.333 \times 10^{-3}$ & $1.121 \times 10^{-2}$ & $7.295 \times 10^{-3}$ \\
\hline
\end{tabular}

Table 27: Percent of confidence ellipsoids which contain the true parameter values $(T=14.14$, constraint implementation $(\mathrm{C} 4))$.

\begin{tabular}{|c||c|c|c|}
\hline & $\sigma_{0}^{2}=0.01$ & $\sigma_{0}^{2}=0.05$ & $\sigma_{0}^{2}=0.10$ \\
\hline \hline$S E$-opt & $94.6 \%(S E=0.71 \%)$ & $93.9 \%(S E=0.76 \%)$ & $95.5 \%(S E=0.66 \%)$ \\
\hline$D$-opt & $94.8 \%(S E=0.70 \%)$ & $94.4 \%(S E=0.73 \%)$ & $94.3 \%(S E=0.73 \%)$ \\
\hline$E$-opt & $94.6 \%(S E=0.71 \%)$ & $94.0 \%(S E=0.75 \%)$ & $93.1 \%(S E=0.80 \%)$ \\
\hline Uniform & $95.2 \%(S E=0.68 \%)$ & $96.3 \%(S E=0.60 \%)$ & $94.5 \%(S E=0.72 \%)$ \\
\hline
\end{tabular}

Table 28: Percent of confidence ellipsoids which contain the true parameter values $(T=28.28$, constraint implementation $(\mathrm{C} 4))$.

\begin{tabular}{|c||c|c|c|}
\hline & $\sigma_{0}^{2}=0.01$ & $\sigma_{0}^{2}=0.05$ & $\sigma_{0}^{2}=0.10$ \\
\hline \hline$S E$-opt & $94.9 \%(S E=0.70 \%)$ & $94.4 \%(S E=0.73 \%)$ & $93.9 \%(S E=0.76 \%)$ \\
\hline$D$-opt & $94.2 \%(S E=0.74 \%)$ & $94.4 \%(S E=0.73 \%)$ & $95.0 \%(S E=0.70 \%)$ \\
\hline$E$-opt & $94.7 \%(S E=0.71 \%)$ & $92.5 \%(S E=0.83 \%)$ & $89.9 \%(S E=0.95 \%)$ \\
\hline Uniform & $96.2 \%(S E=0.60 \%)$ & $94.9 \%(S E=0.70 \%)$ & $94.7 \%(S E=0.71 \%)$ \\
\hline
\end{tabular}




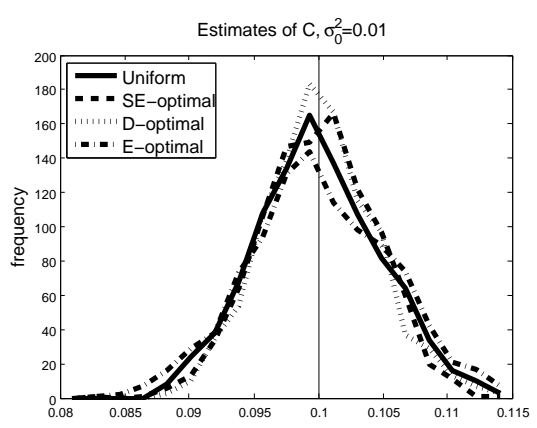

(a)

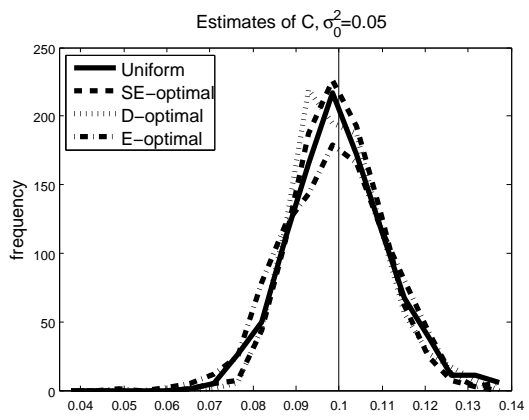

(c)

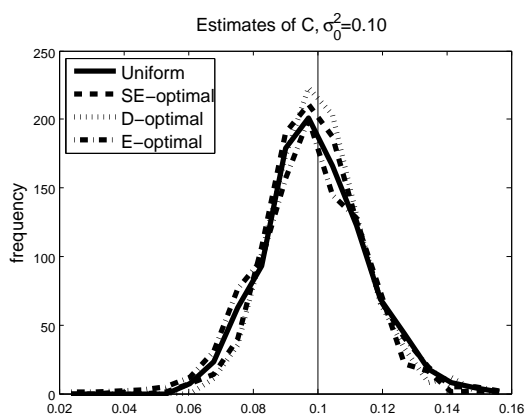

(e)

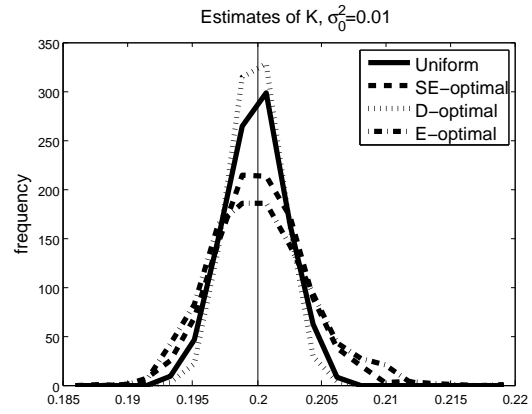

(b)

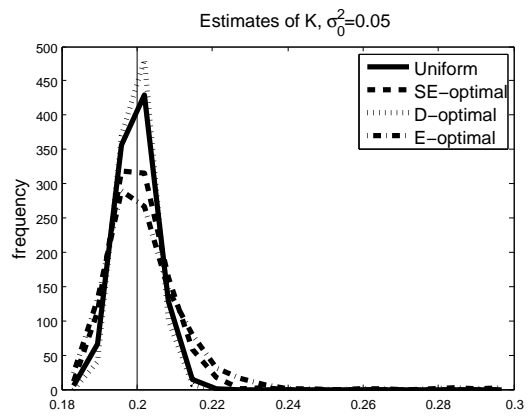

(d)

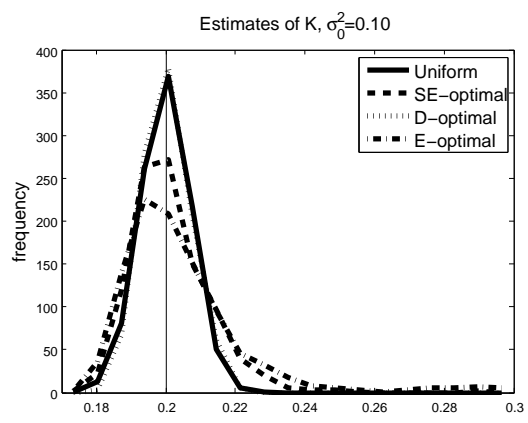

(f)

Figure 18: Histograms of parameter estimates $(\hat{C}$ in panels (a),(c),(e), and $\hat{K}$ in panels (b),(d),(f)) resulting from Monte Carlo simulations. Simulated data was generated with $\sigma_{0}^{2}=0.01$ (top row), $\sigma_{0}^{2}=0.05$ (middle row), and $\sigma_{0}^{2}=0.10$ (bottom row) $(T=28.28$ and constraint implementation $(\mathrm{C} 4))$. 


\section{Concluding Remarks}

We have considered the performance of three optimal design criteria based on the Fisher Information Matrix, the SE-optimal, D-optimal and E-optimal design methods on two popular examples, the logistic growth model and the harmonic oscillator. We use Monte Carlo simulations with $M=1000$ to compare standard errors for estimated parameters with each method. The recently introduced [8] SE-optimal design is quite competitive with and in some cases superior to the more traditional D-optimal and E-optimal design methods. In summary, the results found and reported here are fully consistent with those given in [8] where the analysis is based on asymptotic standard errors computed with a single realization.

\section{Acknowledgements}

This research was supported in part by the National Institute of Allergy and Infectious Diseases under Grant Number R01AI071915-09 and in part by the U.S. Air Force Office of Scientific Research under grant number FA9550-09-1-0226.

\section{References}

[1] A.C. Atkinson and A.N. Donev, Optimum Experimental Designs, Oxford University Press, New York 1992.

[2] H. T. Banks and K. L. Bihari, Modeling and estimating uncertainty in parameter estimation, Inverse Problems, 17 (2001), 95-111.

[3] H.T. Banks, L.W. Botsford, F. Kappel and C. Wang, Modeling and estimation in size structured population models, LCDS/CCS Rep. 87-13, March 1987, Brown Univ.; in Proc. 2nd Course on Math. Ecology, (Trieste, December, 1986), World Scientific Press, Singapore 1988, pp. 521-541.

[4] H.T. Banks, M. Davidian, J.R. Samuels Jr., and K.L. Sutton, An inverse problem statistical methodology summary, CRSC Technical Report CRSC-TR08-01, NCSU, January 2008; Chapter 11 in Statistical Estimation Approaches in Epidemiology, (Gerardo Chowell et al., eds.), Springer, Berlin Heidelberg New York 2009, pp. 249-302.

[5] H.T. Banks, M. Davidian, Shuhua Hu, Grace M. Kepler and E. S. Rosenberg, Modeling HIV immune response and validation with clinical data, CRSC-TR07-09, March 2007; J. Biological Dynamics, 2 (2008), 357-385.

[6] H.T. Banks, J.L. Davis, S.L. Ernstberger, S. Hu, E. Artimovich and A.K. Dhar, Experimental design and estimation of growth rate distributions in size-structured shrimp populations, CRSCTR08-20, November 2008; Inverse Problems, 25 (2009), 095003(28pp).

[7] H.T. Banks, Sava Dediu, S.L. Ernstberger and F. Kappel, A new approach to optimal design problems, CRSC-TR08-12, September, 2008, revised November, 2009; J. Inverse and Ill-posed Problems, 18 (2010), 25-83.

[8] H.T. Banks, K. Holm and F. Kappel, Comparison of optimal design methods in inverse problems, CRSC Technical Report, CRSC-TR10-11, NCSU, Raleigh, July 2010; Inverse Problems, 27 (2011), 075002(31pp). 
[9] H.T. Banks and K. Kunisch, Estimation Techniques for Distributed Parameter Systems, Birkhä user, Boston 1989.

[10] H.T. Banks and H.T. Tran, Mathematical and Experimental Modeling of Physical and Biological Processes, Chapman and Hall/CRC, Boca Raton, FL, 2009.

[11] M.P.F. Berger and W.K. Wong (Editors), Applied Optimal Designs, John Wiley \& Sons, Chichester, UK, 2005.

[12] P. Billingsley, Convergence of Probability Measures, John Wiley \& Sons, New York, NY, 1968.

[13] M. Davidian and D. Giltinan, Nonlinear Models for Repeated Measurement Data, Chapman \& Hall, London, 1998.

[14] V. V. Fedorov, Theory of Optimal Experiments, Academic Press, New York and London, 1972.

[15] V.V. Fedorov and P. Hackel, Model-Oriented Design of Experiments, Springer-Verlag, New York, NY, 1997.

[16] P. J. Huber, Robust Statistics, John Wiley \& Sons, Inc., New York, NY, 1981.

[17] F. Kappel and A. V. Kuntsevich, An implementation of Shor's r-algorithm, Computational Optimization and Applications, 15 (2000), 193-205.

[18] A. Kuntsevich and F. Kappel, SolvOpt, retrieved December 2009, from http://www.kfunigraz.ac.at/imawww/kuntsevich/solvopt/.

[19] W. Müller and M. Stehlik, Issues in the optimal design of computer simulation experiments, Appl. Stochastic Models in Business and Industry, 25 (2009), 163-177.

[20] M. Patan and B. Bogacka, Optimum experimental designs for dynamic systems in the presence of correlated errors, Computational Statistics and Data Analysis, 51 (2007), 5644-5661.

[21] Yu. V. Prohorov, Convergence of random processes and limit theorems in probability theory, Theor. Prob. Appl., 1 (1956), 157-214.

[22] F. Pukelsheim, Optimal Design of Experiments, JohnWiley \& Sons, New York, NY, 1993.

[23] G.A.F. Seber and C.J. Wild, Nonlinear Regression, Wiley-Interscience, Hoboken, 2003.

[24] D. Ucinski and A.C. Atkinson, Experimental design for time-dependent models with correlated observations, Studies in Nonlinear Dynamics and Econometrics, 8(2) (2004), Article 13: The Berkeley Electronic Press. 